\title{
Damage Detection of Laminated Rubber Bearings Based on the Antiresonance Frequencies of Periodic Structure and Its Sensitivity Analysis
}

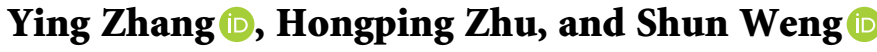 \\ School of Civil \& Hydraulic Engineering, Huazhong University of Science and Technology, Wuhan 430074, China \\ Correspondence should be addressed to Shun Weng; wengshun@mail.hust.edu.cn
}

Received 21 April 2021; Accepted 25 November 2021; Published 15 January 2022

Academic Editor: Luis A. Felipe-Sese

Copyright (c) 2022 Ying Zhang et al. This is an open access article distributed under the Creative Commons Attribution License, which permits unrestricted use, distribution, and reproduction in any medium, provided the original work is properly cited.

\begin{abstract}
An isolation bearing consumes most of the seismic energy of a structure and is vulnerable to destruction. The performance of isolation bearings is usually evaluated according to the global stiffness and energy dissipation capacity. However, the early minor damage in isolation bearings is difficult to identify. In this study, a damage detection scheme for the isolation bearing is proposed by focusing on the antiresonance of the quasiperiodic structure. Firstly, a laminated rubber bearing was simplified as a monocoupled periodic rubber-steel structure. The characteristic equation of the driving point antiresonance frequency of the periodic system was achieved via the dynamic stiffness method. Secondly, the sensitivity coefficient of the driving point antiresonance, which was obtained from the first-order derivative of the antiresonance frequency, with respect to the damage scaling parameter was derived using the antiresonance frequency characteristic equation. Thirdly, the optimised driving points of the antiresonance frequencies were selected by means of sensitivity analysis. Finally, from the measured changes in the antiresonance frequencies, the damage was identified by solving the sensitivity identification equation via a numerical optimisation method. The application of the proposed method to laminated rubber bearings under various damage cases demonstrates the feasibility of this method. This study has proven that changes in the shear modulus of each rubber layer can be identified accurately.
\end{abstract}

\section{Introduction}

During an earthquake disaster, the seismic isolator consumes a large amount of structural vibration energy. The isolator is the most vulnerable part of the structure that is expected to be destroyed. If the early damage of the seismic isolator cannot be identified and dealt with in time, then it will continue to accumulate and may lead to the sudden structural failure. However, the early minor damage in the isolation bearing cannot be reflected from the test results of mechanical properties. Therefore, the effective monitoring and evaluation of the seismic isolator is of great significance.

The laminated rubber bearing is widely used in seismic isolation structures. Most theoretical studies have investigated the behaviour of laminated rubber bearings in terms of Haringx's theory $[1,2]$. In the model of Haringx's equivalent column, the rubber layers and steel shims are treated as homogeneous and isotropic materials. For moderate amounts of shear strain, horizontal stiffness of the elastomeric bearing in Haringx's theory agrees with the experimental results $[3,4]$. Haringx's theory has been applied in the investigation of the stability of rubber bearings [5-8]. On the basis of Haringx's theory, Koh and Kelly [9] proposed a nonlinear mechanical model to predict the horizontal displacement of the elastomeric bearing under large axial force, and the experimental results of Buckle et al. [10] validated this model. The limitations of the investigations lie in the inability to address the details of the mechanical behaviours of interlaminated rubber layers [4], and the traditional approach cannot handle the influence of geometric and material parameters of each rubber layer. In addition, according to the results of finite element analysis, Kalfas et al. $[11,12]$ found the damage magnitude of each rubber layer and steel shim is different after the bearing under a certain 
load. When Haringx's theory is applied to the damage identification of laminated rubber bearings, the damage of certain layers cannot be located; consequently, the early minor damage in the bearings is difficult to identify.

The periodical model can be used to analyse the internal deformation and force of individual cells. Therefore, the laminated rubber bearing model that considers the periodicity can be used to identify the damage of each layer. As a typical one is consisting of several alternating layers of rubber bonded to interleaving steel shims [13]; it can be considered a chain-like periodic structure system composed of an end-to-end repeating structure. Static models $[4,14,15]$ and dynamic models $[13,16]$ of laminated rubber bearing that consider periodicity have been developed. However, the dynamic characteristics will change when damage occurs in a periodic structure.

Dynamic characteristics, such as natural frequencies and mode shapes, have been used in the damage detection of periodic structures [17-19]. Zhu [17] used the characteristic receptance approach to obtain the frequency characteristic equation of a periodic spring-mass system with a single damage and identified the damage of it based on the sensitivity of natural frequencies. This method can be applied to various types of finite monocoupled periodic systems with multiple damage [17]. To extend the work [17], Yin [18] used natural frequencies to identify the damage of periodically supported structures. Zhu [19] used the slopes and curvatures of mode shapes to localise damage, which were then quantified using natural frequencies. The abovementioned studies showed that the damage of periodic structures can be identified by using natural frequencies and mode shapes.

On the one hand, natural frequencies are less sensitive to local damage compared with mode shapes [19]. On the other hand, the mode shapes have generally lower accuracy than natural frequencies during measurements. In addition, as the total number of natural frequencies is always limited in high-quality measurements [20], the available modal data that can be used in damage detection is usually incomplete. Natural frequencies and mode shapes are clearly inadequate in damage identification; thus, antiresonance frequencies have been applied to the finite element model updating and damage identification of structures [21-27]. Dilena [22] found that the appropriate use of natural frequencies and antiresonance frequencies could avoid the nonuniqueness of the damage location problem, which occurs in symmetrical beams when only frequency data are employed. Wang [23] utilised the first antiresonance to localise the crack of a Timoshenko beam. Hanson [24] used antiresonance frequencies to update the model of a two-degrees-of-freedom (DOF) system. Sinou [25] detected the breathing crack of a pipeline beam based on the antiresonances of higher-order frequency response functions (FRFs). In an experimental study on the crack identification of thick beams, Hou [26] proved that the incorporation of antiresonance frequencies could enhance the modal dataset in finite element model updating. Another study involving model updating found that antiresonance frequencies could be used as an alternative of natural frequencies and mode shapes [27].
Antiresonance frequencies reflect not only the intrinsic characteristics of structures but also their local physical characteristics. Therefore, the antiresonance frequency is further extended to the damage identification of periodic structures in this study.

Two methods are generally used to determine antiresonance frequencies. The first method is to extract data from the frequency response or impedance curve [23-26], which are the zeros of the curves. The other method is to calculate the eigenvalues of a structure under a variety of artificial constraints applied to the ground combined with transducer location data [28,29]. A set of sensitivities of antiresonance frequencies [28] was used for damage identification. According to Mottershead [28], the sensitivity of an antiresonance frequency is a linear combination of the sensitivity of mode shapes at the same point and the sensitivity of natural frequencies. However, the calculation of antiresonance frequencies and its sensitivity of periodic structures has not yet been studied. As for the periodic structure, each cell is the same, which implies that the periodicity of the structure can be exploited to simplify the analytic process.

At present, the damage detection of laminated rubber bearing based on the antiresonance frequencies of periodic structure is at an early stage. According to the damage detection method for laminated rubber bearing based on the characteristic receptance method developed by Zhang [30], only single damage can be identified. And the influence of driving points on identification results has not been studied. However, as each node of the structure can be configured as the driving point, additional appropriate driving points should be selected to be able to extract the antiresonance frequencies and improve the modal identification. Antiresonance frequencies can be obtained from the point FRFs and transfer FRFs, in which the former is more robust than the latter [21]. Mao [29] studied the selection of optimal antiresonance frequencies but did not distinguish the driving point antiresonance from the transfer point antiresonance. Therefore, the selection of the optimal driving point is investigated in this study.

This study proposes a damage detection method of laminated bearings in service based on the sensitivity analysis of the antiresonance frequency of periodic structures. Firstly, the laminated rubber bearing is modelled as a monocoupled periodic rubber-steel structure. The antiresonance frequency of the periodic structure is derived using Bloch's theorem and dynamic stiffness method. Secondly, the relationship between the antiresonance frequency and damage scaling parameter is derived according to the antiresonance frequency characteristic equation. From the results of the sensitivity analysis, more suitable driving points of the antiresonance frequencies are selected and used in damage identification. Finally, by taking the antiresonance frequencies before and after the damage as the objective function, the sensitivity identification equation is solved via a numerical optimisation method. By detecting the simulated damage of one or more parts of the laminated rubber bearing, the accuracy of the proposed method was verified. 


\section{Antiresonance Frequency Characteristic Equation of the Periodic Rubber- Steel Structure}

The laminated rubber bearing is considered a periodic rubber-steel structure, as shown in Figure 1(a). Each periodic cell consists of a rubber layer and two steel shims, as depicted in Figure 1(b). Figure 2 illustrates a monocoupled $N_{P}$-cell periodic structure terminating at the fixed end $O$ and the free end $N$.

In the horizontal direction, the steel plates do not affect the shear deformations of the rubber layers in the bearing. Since the ratio of the shear modulus of steel to that of rubber is greater than $1 \times 10^{4}$, the deformation of the steel shim is negligible in the analysis. Therefore, the deformation of the isolation bearing is considered to be entirely caused by the rubber layer. The rubber layer is simplified as a shear beam. Here, only the shear deformation is considered, and the bending deformation is neglected. The parameters of the rubber layer are as follows: the thickness of each layer is given by $l$, the cross-sectional area is given by $A$, the shear modulus is given by $G$, and the density is given by $\rho$. The stiffness and mass matrix of the rubber layer are as follows:

$$
\begin{aligned}
& \mathbf{k}_{r}=\int_{0}^{l} G A\left[\frac{\mathrm{d} \mathbf{N}_{w}}{\mathrm{~d} x}\right]^{T}\left[\frac{\mathrm{d} \mathbf{N}_{w}}{\mathrm{~d} x}\right] \mathrm{d} x=\frac{G A}{l}\left[\begin{array}{cc}
1 & -1 \\
-1 & 1
\end{array}\right], \\
& \mathbf{m}_{r}=\int_{0}^{l} \rho A\left[\mathbf{N}_{w}\right]^{T}\left[\mathbf{N}_{w}\right] \mathrm{d} x=\frac{\rho A l}{6}\left[\begin{array}{ll}
2 & 1 \\
1 & 2
\end{array}\right],
\end{aligned}
$$

where $N_{w}(x)=\left[\begin{array}{lll}1-x / l & x / l\end{array}\right]$ is the shape function vector.
The lumped mass is used for describing the steel shim. The mass of each steel shim in the symmetrical cell is calculated as $m_{s} / 2$. With the introduction of the mass ratio of steel to rubber, $\theta=m_{s} / m_{r}$, where $m_{r}=\rho A l$ is the mass of each rubber layer. The mass matrix of the composite cell is given by

$$
\mathbf{m}=\mathbf{m}_{r}+\mathbf{m}_{s}=\left[\begin{array}{cc}
\frac{\rho A l}{3}+\frac{m_{s}}{2} & \frac{\rho A l}{6} \\
\frac{\rho A l}{6} & \frac{\rho A l}{3}+\frac{m_{s}}{2}
\end{array}\right]=\rho A l\left[\begin{array}{cc}
\frac{1}{3}+\frac{\theta}{2} & \frac{1}{6} \\
\frac{1}{6} & \frac{1}{3}+\frac{\theta}{2}
\end{array}\right] .
$$

When the structure vibrates harmonically with angular frequency $\omega$, the dynamic stiffness matrix of the composite periodic cell can be expressed as

$$
\mathbf{d}=\mathbf{k}_{r}-\omega^{2} \mathbf{m}=\left[\begin{array}{ll}
d_{L L} & d_{L R} \\
d_{R L} & d_{R R}
\end{array}\right],
$$

where

$$
\begin{aligned}
& d_{L L}=d_{R R}=\frac{G A}{l}-\omega^{2}\left(\frac{1}{3}+\frac{\theta}{2}\right) \rho A l, \\
& d_{L R}=d_{R L}=-\frac{G A}{l}-\omega^{2} \frac{\rho A l}{6} .
\end{aligned}
$$

For a monocoupled $N_{P}$-cell periodic structure with one fixed end and one free end, the global dynamic stiffness matrix can be expressed as
For an undamped structure, the FRFs of a driving point, i.e., $j$-th node, can be expressed as

$$
H_{j}=\frac{\operatorname{det}(\mathbf{D})_{j}}{\operatorname{det}(\mathbf{D})} .
$$

$\operatorname{det}(\cdot)$ represents the determinant of the matrix $\mathbf{D}$, and subscript $j$ means that the $j$-th row and the $j$-th column are deleted. For the driving point FRFs of an undamped structure, the antiresonance frequencies $z_{j}$ are the zeros of the numerator polynomial [27], which satisfies

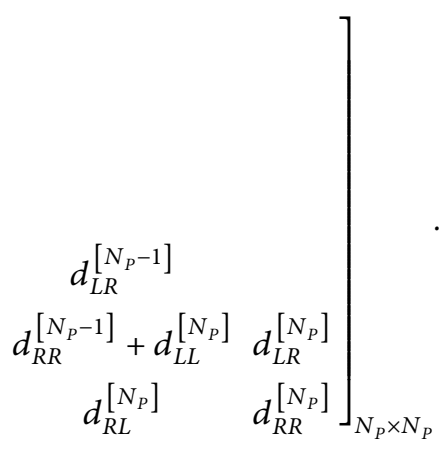

$$
\operatorname{det}(\mathbf{D})_{j}=0
$$

The determinant $\operatorname{det}(\mathbf{D})_{j}$ can be rewritten as the determinant of a partitioned matrix as

$$
\operatorname{det}(\mathbf{D})_{j}=\left|\begin{array}{cc}
\mathbf{D}_{L} & 0 \\
0 & \mathbf{D}_{R}
\end{array}\right|=\left|\mathbf{D}_{L}\right| \cdot\left|\mathbf{D}_{R}\right|,
$$

where $\left|\mathbf{D}_{L}\right|$ and $\left|\mathbf{D}_{\mathrm{R}}\right|$ are the characteristic determinants of the substructures on both sides of the driving point, and the detailed expressions of $\left|\mathbf{D}_{\mathrm{L}}\right|$ and $\left|\mathbf{D}_{\mathrm{R}}\right|$ are provided in Appendix. 


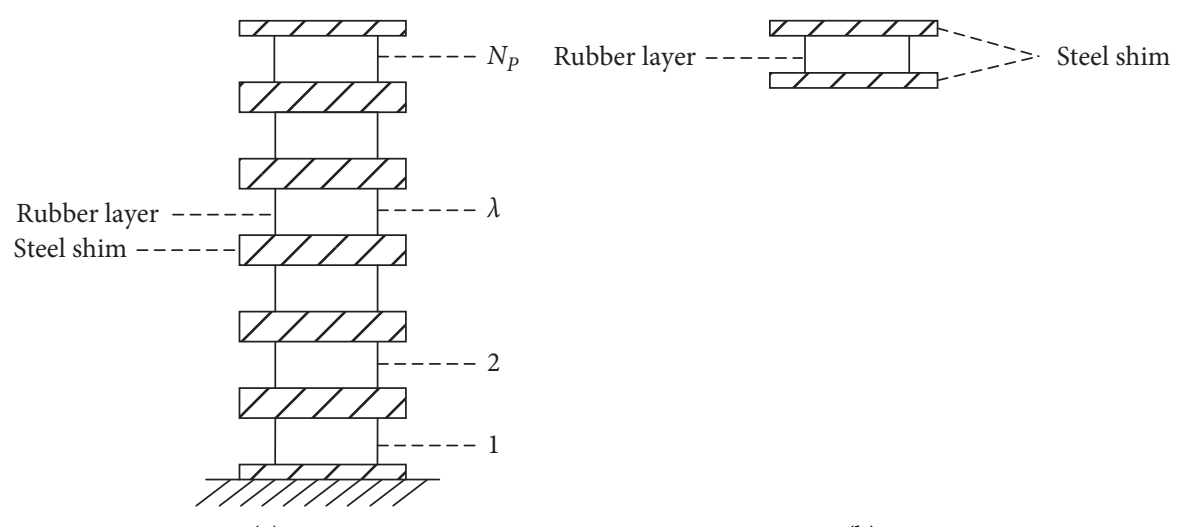

(a)

(b)

Figure 1: (a): The model of a laminated rubber bearing. (b): Rubber-steel cell.

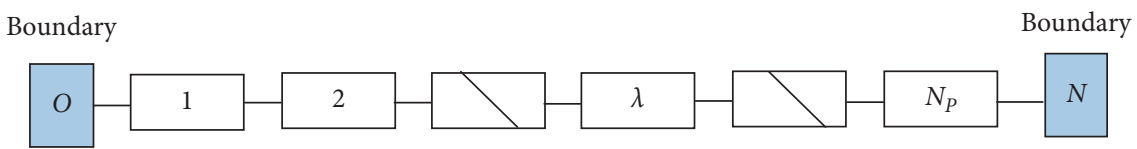

FIgURE 2: The monocoupled periodic structure of $N_{P}$ cells with a free end $(\mathrm{N})$ and a fixed end (O).

By substituting equation (7) into (6), the characteristic equation of the driving point antiresonance frequencies can be obtained.

$$
\left|\mathbf{D}_{L}\right| \cdot\left|\mathbf{D}_{R}\right|=0 .
$$

As shown in equation (8), antiresonance occurs provided that the driving frequency reaches a natural frequency of the substructure on the left or right side of the driving point. $\left|\mathbf{D}_{L}\right|=0$ gives the characteristic equation of periodic structure 1 consisting of $j$ cells with two fixed ends. $\left|\mathbf{D}_{R}\right|=0$ gives the characteristic equation of periodic structure 2 consisting of $\left(N_{P}-j\right)$ cells and with one free end and one fixed end. In other words, the antiresonance frequencies reflect the natural frequencies of the substructure on either side of the driving point.

For a perfectly periodic structure, the dynamic stiffness matrix of each cell is exactly the same. Take the $\lambda$-th $(\lambda=1,2$, $\ldots, N_{P}$ ) cell as an example. The displacements and forces at the ends of the cell can be expressed in relation to the dynamic stiffness matrix as follows:

$$
\left[\begin{array}{ll}
d_{L L} & d_{L R} \\
d_{R L} & d_{R R}
\end{array}\right]\left\{\begin{array}{c}
X_{L}^{[\lambda]} \\
X_{R}^{[\lambda]}
\end{array}\right\}=\left\{\begin{array}{c}
F_{L}^{[\lambda]} \\
F_{R}^{[\lambda]}
\end{array}\right\} .
$$

In terms of the vibration of the monocoupled periodic structure, according to Bloch's theorem, the state vector at the left end of a cell must be equal to $e^{\mu}$ times the state vector at the left end of the next cell, where $\mu$ is the propagation constant $[31,32]$. The relationship between the displacements and forces of the two adjacent cells can be determined in relation to the propagation constant $\mu$.

$$
\left\{\begin{array}{c}
X_{L}^{[\lambda+1]} \\
F_{L}^{[\lambda+1]}
\end{array}\right\}=e^{-\mu}\left\{\begin{array}{c}
X_{L}^{[\lambda]} \\
F_{L}^{[\lambda]}
\end{array}\right\},
$$

where the superscript $\lambda$ and $\lambda+1$ refer to cell $\lambda$ and the next cell $\lambda+1$.

The displacements and equilibrium of the forces at the interface of the $\lambda$-th cell and the $(\lambda+1)$-th cell are compatible. Thus,

$$
\left\{\begin{array}{l}
X_{L}^{[\lambda+1]} \\
F_{L}^{[\lambda+1]}
\end{array}\right\}=\left[\begin{array}{cc}
1 & 0 \\
0 & -1
\end{array}\right]\left\{\begin{array}{c}
X_{R}^{[\lambda]} \\
F_{R}^{[\lambda]}
\end{array}\right\} .
$$

By combining equations (10) and (11), the displacements and forces at the right and left ends of the $\lambda$-th cell become related to the propagation constant $\mu$.

$$
\left\{\begin{array}{c}
X_{R}^{[\lambda]} \\
F_{R}^{[\lambda]}
\end{array}\right\}=\left[\begin{array}{cc}
e^{-\mu} & 0 \\
0 & -e^{-\mu}
\end{array}\right]\left\{\begin{array}{c}
X_{L}^{[\lambda]} \\
F_{L}^{[\lambda]}
\end{array}\right\} .
$$

A periodic structure with symmetric cells has $d_{L L}=d_{R R}$ and $d_{L R}=d_{R L}$. Substituting equation (11) into (9) yields the expression of the propagation constant $\mu$.

$$
\cosh \mu=-\frac{d_{L L}}{d_{L R}}
$$

For the perfectly periodic structure, the determinant $\left|\mathbf{D}_{L}\right|$ in equation (A.1a) can be expressed by the dynamic stiffness of any cell. When the dynamic stiffness of the $\lambda$-th $(\lambda=1,2, \ldots, j)$ cell, i.e., $d_{L L}^{[\lambda]}$ and $d_{L R}^{[\lambda]}$, is reserved, $\left|\mathbf{D}_{L}\right|$ can be rewritten using equation (13) as follows: 


$$
\left|\mathbf{D}_{L}\right|=\left(-d_{L R}\right)^{j-1}\left|\overline{\mathbf{D}}_{L}\right|,
$$

where $\left|\bar{D}_{\mathrm{L}}\right|$ is the determinant of order $(j-1)$,

$$
\left|\overline{\mathbf{D}}_{L}\right|=\left[\begin{array}{c}
\frac{\left(d_{L L}^{[\lambda]}\right)^{2}-\left(d_{L R}^{[\lambda]}\right)^{2}}{\left(d_{L R}\right)^{2}} \frac{\sinh (\lambda-1) \mu \sinh (j-\lambda) \mu}{\sinh ^{2} \mu} \\
-\frac{d_{L L}^{[\lambda]}}{d_{L R}} \frac{\sinh (j-1) \mu}{\sinh \mu}+\cosh (j-\lambda) \mu \cos h(\lambda-1) \mu
\end{array}\right] .
$$

Furthermore, by setting $d_{L L}^{[\lambda]}=d_{L L}$ and $d_{L R}^{[\lambda]}=d_{L R}$ in equation (14b), $\left|\bar{D}_{\mathrm{L}}\right|$ can be rewritten as

$$
\left|\overline{\mathbf{D}}_{L}\right|=\frac{\sinh (j \mu)}{\sinh \mu} .
$$

Similarly, the determinant $\left|\mathbf{D}_{\mathrm{R}}\right|$ in equation (A.1 b) can be expressed by the dynamic stiffness of the $\lambda$-th $(\lambda=j+1$, $\left.j+2, \ldots, N_{P}\right)$ cell,

$$
\left|\mathbf{D}_{R}\right|=\left(-d_{L R}\right)^{N_{P}-j}\left|\overline{\mathbf{D}}_{R}\right|,
$$

where $\left|\bar{D}_{\mathrm{R}}\right|$ is the determinant of order $\left(N_{P}-j\right)$,

$$
\left|\overline{\mathbf{D}}_{R}\right|=\left[\begin{array}{c}
\frac{\left(d_{L L}^{[\lambda]}\right)^{2}-\left(d_{L R}^{[\lambda]}\right)^{2}}{\left(d_{L R}\right)^{2}} \frac{\sinh (\lambda-j-1) \mu \sinh \left(N_{P}-\lambda\right) \mu}{\sinh \mu} \\
-\frac{d_{L L}^{[\lambda]}}{d_{L R}} \cosh \left(N_{P}-j-1\right) \mu+\cosh (\lambda-j-1) \mu \sinh \left(N_{P}-\lambda\right) \mu \sinh \mu
\end{array}\right]=\cosh \left[\left(N_{P}-j\right) \mu\right]
$$

The detailed calculation process of determinants $\left|\bar{D}_{\mathrm{L}}\right|$ and $\left|\bar{D}_{\mathrm{R}}\right|$ are provided in Appendix.

By substituting equations (14) and (15) into (8), the characteristic equation of the driving point antiresonance frequencies of the periodic structure containing the information of the location of different cells can be obtained.

$$
\begin{gathered}
\cosh \left(N_{P}-j\right) \mu_{z}\left[\begin{array}{c}
\frac{\left(d_{L L}^{[\lambda]}\right)^{2}-\left(d_{L R}^{[\lambda]}\right)^{2}}{\left(d_{L R}\right)^{2}} \frac{\sinh (\lambda-1) \mu_{z} \sinh (j-\lambda) \mu_{z}}{\sin h^{2} \mu_{z}} \\
-\frac{d_{L L}^{[\lambda]}}{d_{L R}} \frac{\sinh (j-1) \mu_{z}}{\sin h \mu_{z}}+\cosh (\lambda-1) \mu_{z} \cosh (j-\lambda) \mu_{z}
\end{array}\right]=0 \\
\sinh \left(j \mu_{z}\right)\left[\begin{array}{c}
\frac{\left(d_{L L}^{[\lambda]}\right)^{2}-\left(d_{L R}^{[\lambda]}\right)^{2}}{\left(d_{L R}\right)^{2}} \frac{\sin h\left(N_{P}-\lambda\right) \mu_{z} \sin h(\lambda-j-1) \mu_{z}}{\sin h^{2} \mu_{z}} \\
-\frac{d_{L L}^{[\lambda]}}{d_{L R}} \frac{\cosh \left(N_{P}-j-1\right) \mu_{z}}{\sin h \mu_{z}}+\sin h\left(N_{P}-\lambda\right) \mu_{z} \cosh (\lambda-j-1) \mu_{z}
\end{array}\right]=0, \\
\quad\left(\lambda=j+1, j+2, \ldots, N_{P}\right) .
\end{gathered}
$$

The propagation constant $\mu$ and arbitrary vibration frequency $\omega$ have a one-to-one correspondence. Here, $\mu_{z}$ denotes the propagation constant corresponding to the antiresonance frequency $z_{j}$. The characteristic equation of the driving point antiresonance frequencies of the periodic structure expressed as the propagation constant can be obtained using

$$
\sinh \left(j \mu_{z}\right) \cosh \left(N_{P}-j\right) \mu_{z}=0 .
$$

When the vibration frequency $\omega$ is equal to the antiresonance frequency $z_{j}$, combining equations (3) and (13) yields the relationship between the propagation constant $\mu_{z}$ and antiresonance frequency $z_{j}$.

$$
\cosh \mu_{z}=\frac{6 G-z_{j}^{2}(2+3 \theta) \rho l^{2}}{6 G+z_{j}^{2} \rho l^{2}} .
$$

The propagation constant is usually written in complex form, i.e., $\mu=\delta+i \gamma$, where ' $i$ ' is the imaginary unit, namely, 
$\mathrm{i}=\sqrt{-1}$. The real part $\delta$ represents the attenuation constant, whereas the imaginary part $\gamma$ represents the phase constant [33]. When $\delta \neq 0$, the wave is in a frequency stopband; when $\delta=0$, the wave is in a passband [34]. For an undamped periodic structure with symmetric cells, the natural frequencies lie within the passband [35]. In addition, the driving point antiresonance frequencies interlace the natural frequencies [21]. Therefore, the driving point antiresonance frequencies also lie within the passband (i.e., $\delta=0$ ). Substituting $\mu=\mathrm{i} \gamma$ and equation (18) into (16) yields the antiresonance frequencies of the periodic rubber-steel structure in the healthy status.

$$
z_{j}^{u}=2 \sin \frac{\gamma^{u}}{2} \sqrt{\frac{3 G}{\left(\cos \gamma^{u}+2+3 \theta\right) \rho l^{2}}},
$$

where $\quad \gamma^{u}=\gamma_{L}^{u}=\pi k_{1} / j\left(k_{1}=1,2, \ldots, j-1\right)$ or $\gamma^{u}=\gamma_{R}^{u}=$ $(\pi / 2)\left(2 k_{2}-1 / N_{P}-j\right)\left(k_{2}=1,2, \ldots, N_{P}-j\right)$, in which the superscript ' $u$ ' means undamaged.

\section{Sensitivity Analysis}

3.1. Sensitivity of Driving Point Antiresonance. When the shear modulus of the $\lambda$-th $\left(\lambda=1,2, \ldots, N_{P}\right)$ cell in the periodic structure changes slightly, the first-order Taylor's expansion of the antiresonance frequency of driving point $j$ is

$$
z_{j}\left(G+\Delta G^{[\lambda]}\right)=z_{j}(G)+\frac{\partial z_{j}}{\partial G^{[\lambda]}} \Delta G^{[\lambda]},
$$

where the first-order partial derivate $\partial z_{j} / \partial G^{[\lambda]}$ is the sensitivity coefficient of the antiresonance frequency with respect to the shear modulus of the $\lambda$-th $\left(\lambda=1,2, \ldots, N_{P}\right)$ cell.

According to the characteristic equation of the driving point antiresonance frequencies, i.e., equation (16), the sensitivity coefficient of the antiresonance frequency with respect to the shear modulus of the $\lambda$-th cell is as follows:

$$
\frac{\partial z_{j}}{\partial G^{[\lambda]}}=-\frac{\Gamma\left(z_{j}, G^{[\lambda]}\right) / \partial G^{[\lambda]}}{\Gamma\left(z_{j}, G^{[\lambda]}\right) / \partial z_{j}},
$$

where the function $\Gamma\left(z_{j}, G^{[\lambda]}\right)$ is set according to equation (16), i.e.,

$$
\begin{gathered}
\Gamma\left(z_{j}, G^{[\lambda]}\right)=\cosh \left(N_{P}-j\right) \mu_{z}\left[\begin{array}{c}
\frac{\left(d_{L L}^{[\lambda]}\right)^{2}-\left(d_{L R}^{[\lambda]}\right)^{2}}{\left(d_{L R}\right)^{2}} \frac{\sinh (\lambda-1) \mu_{z} \sinh (j-\lambda) \mu_{z}}{\sinh h_{z} \mu_{z}} \\
-\frac{d_{L L}^{[\lambda]}}{d_{L R}} \frac{\sinh (j-1) \mu_{z}}{\sinh \mu_{z}}+\cosh (\lambda-1) \mu_{z} \cosh (j-\lambda) \mu_{z}
\end{array}\right], \quad(\lambda=1,2, \ldots, j), \\
\Gamma\left(z_{j}, G^{[\lambda]}\right)=\sinh \left(j \mu_{z}\right)\left[\begin{array}{c}
\frac{\left(d_{L L}^{[\lambda]}\right)^{2}-\left(d_{L R}^{[\lambda]}\right)^{2}}{\left(d_{L R}\right)^{2}} \frac{\sinh (\lambda-j-1) \mu_{z} \sinh \left(N_{P}-\lambda\right) \mu_{z}}{\sin h^{2} \mu_{z}} \\
-\frac{d_{L L}^{[\lambda]}}{d_{L R}} \frac{\cosh \left(N_{P}-j-1\right) \mu_{z}}{\sinh \mu_{z}}+\sinh \left(N_{P}-\lambda\right) \mu_{z} \cosh (\lambda-j-1) \mu_{z}
\end{array}\right], \\
\left(\lambda=j+1, j+2, \ldots, N_{P}\right) .
\end{gathered}
$$

The first partial derivatives $\partial \Gamma\left(z_{j}, G^{[\lambda]}\right) / \partial G^{[\lambda]}$ and $\partial \Gamma\left(z_{j}, G^{[\lambda]}\right) / \partial z_{j}$ in equation (21) can be obtained from (22), which contains the information about the location of the different cells.

$$
\begin{gathered}
\frac{\partial \Gamma\left(z_{j}, G^{[\lambda]}\right)}{\partial z_{j}}=\frac{\left(N_{P}-j\right) \sinh \left(N_{P}-j\right) \mu_{z} \sinh \left(j \mu_{z}\right)+j \cosh \left(N_{P}-j\right) \mu_{z} \cosh \left(j \mu_{z}\right)}{\sinh \mu_{z}} \frac{\partial \mu_{z}}{\partial z_{j}}, \\
\frac{\partial \Gamma\left(z_{j}, G^{[\lambda]}\right)}{\partial G^{[\lambda]}}=-\frac{\cosh \left(N_{P}-j\right) \mu_{z}}{d_{L R} \sinh ^{2} \mu_{z}}\left[\begin{array}{c}
2\left(\cosh \mu_{z} \frac{\partial d_{L L}^{[\lambda]}}{\partial G^{[\lambda]}}+\frac{\partial d_{L R}^{[\lambda]}}{\partial G^{[\lambda]}}\right) \sinh (j-\lambda) \mu_{z} \sinh (\lambda-1) \mu_{z} \\
+\frac{\partial d_{L L}^{[\lambda]}}{\partial G^{[\lambda]}} \sinh \mu_{z} \sinh (j-1) \mu_{z} \quad
\end{array}\right] \\
\quad(\lambda=1,2, \ldots, j),
\end{gathered}
$$




$$
\frac{\partial \Gamma\left(z_{j}, G^{[\lambda]}\right)}{\partial G^{[\lambda]}}=-\frac{\sinh \left(j \mu_{z}\right)}{d_{L R} \sinh \mu^{2} \mu_{z}}\left[\begin{array}{c}
2\left(\cosh \mu_{z} \frac{\partial d_{L L}^{[\lambda]}}{\partial G^{[\lambda]}}+\frac{\partial d_{L R}^{[\lambda]}}{\partial G^{[\lambda]}}\right) \sinh (\lambda-j-1) \mu_{z} \sinh \left(N_{P}-\lambda\right) \mu_{z} \\
+\frac{\partial d_{L L}^{[\lambda]}}{\partial G^{[\lambda]}} \sinh \mu_{z} \cosh \left(N_{P}-j-1\right) \mu_{z}
\end{array}\right] .
$$

The detailed expressions of $\partial d_{L L}^{[\lambda]} / \partial G^{[\lambda]}, \partial d_{L R}^{[\lambda]} / \partial G^{[\lambda]}$, and $\partial \mu_{z} / \partial z_{j}$ in equation (23) can be obtained from equations (3) and (18):

$$
\begin{aligned}
& \frac{\partial d_{L L}^{[\lambda]}}{\partial G^{[\lambda]}}=\frac{A}{l}, \\
& \frac{\partial d_{L R}^{[\lambda]}}{\partial G^{[\lambda]}}=-\frac{A}{l},
\end{aligned}
$$

$$
\frac{\partial \mu_{z}}{\partial z_{j}}=\frac{\left(2+3 \theta+\cosh \mu_{z}\right) \rho A l z_{j}}{3 d_{L R} \sinh \mu_{z}}
$$

where $l$ is the thickness of each layer, $A$ is the cross-sectional area, and $\theta$ is the mass ratio of steel to rubber.

Combining equations (19), (21), (23), and (24) yields the sensitivity coefficient $\partial z_{j} / \partial G^{[\lambda]}$.

$$
\begin{aligned}
& \frac{\partial z_{j}}{\partial G^{[\lambda]}}= \begin{cases}\frac{2}{j} \sqrt{\frac{3}{\left(\cos \gamma^{u}+2+3 \varphi\right) G \rho l^{2}}} \sin \frac{\gamma^{u}}{2} \cos ^{2}\left(\frac{2 \lambda-1}{2} \gamma^{u}\right), & (\lambda=1,2, \ldots, j), \\
0, & \left(\lambda=j+1, j+2, \ldots, N_{P}\right) .\end{cases} \\
& \left(\gamma^{u}=\gamma_{L}^{u}=\pi \frac{k_{1}}{j}, \quad k_{1}=1,2, \ldots, j-1\right), \\
& \frac{\partial z_{j}}{\partial G^{[\lambda]}}= \begin{cases}0, & (\lambda=1,2, \ldots, j) \\
\frac{2}{N_{P}-j} \sqrt{\frac{3}{\left(\cos \gamma^{u}+2+3 \theta\right) G \rho l^{2}}} \sin \frac{\gamma^{\mathrm{u}}}{2} \cos ^{2}\left(\frac{2 \lambda-2 j-1}{2} \gamma^{u}\right), \quad\left(\lambda=j+1, \quad j+2, \ldots, N_{P}\right)\end{cases} \\
& \left(\gamma^{\mathrm{u}}=\gamma_{\mathrm{R}}^{\mathrm{u}}=\frac{\pi}{2} \frac{2 k_{2}-1}{N_{P}-j}, \quad k_{2}=1,2, \ldots, N_{P}-j\right) .
\end{aligned}
$$

As shown in equation (25), the sensitivity coefficient $\partial z_{j} / \partial G^{[\lambda]}$ is dependent on the total number of periodic cells $N_{P}$, the driving point $j$, the location of the cell $\lambda$, the propagation constant corresponding to the antiresonance frequency $\gamma^{u}$, the mass ratio $\theta$, the shear modulus $G$, the thickness $l$, and the density $\rho$ of the rubber layer. Each cell $\lambda$ has a specific sensitivity coefficient $\partial z_{j} / \partial G^{[\lambda]}$ that can be used for damage identification.

\subsection{Selection of Optimal Driving Points of Antiresonance for} Damage Identification. Given that a structure comprises more than one driving point, the amount of data of antiresonance frequencies is larger than the amount of data of natural frequencies. Besides, in the real practice of realising damage identification, sufficient structural information can be obtained by selecting a small number of driving points. Therefore, the optimal driving point should be selected.

In this section, the optimal driving points are selected by conducting a sensitivity analysis of the antiresonance frequency on the location of driving point $j$. Supposing the thickness of the steel shim is neglected, the length of each periodic cell denoted by $l$ is equal to the thickness of the rubber layer. Thus, the location of driving point $j$ can be expressed as

$$
x_{j}=j l\left(j=1,2, \ldots, N_{P}\right) .
$$

By substituting equation (26) into (19), the antiresonance frequency can be rewritten as 


$$
\begin{aligned}
& z_{j}^{u}=2 \sin \frac{\pi k_{1} l}{2 x_{j}} \sqrt{\frac{3 G}{\left(\cos \left(\pi k_{1} l / x_{j}\right)+2+3 \theta\right) \rho l^{2}}}\left(k_{1}=1,2, \ldots, j-1\right) \\
& z_{j}^{u}=2 \sin \frac{\pi\left(2 k_{2}-1\right) l}{4\left(N_{P} l-x_{j}\right)} \sqrt{\frac{3 G}{\left[\cos \left(\pi\left(2 k_{2}-1\right) l / 2\left(N_{P} l-x_{j}\right)\right)+2+3 \theta\right] \rho l^{2}}}\left(k_{2}=1,2, \ldots, N_{P}-j\right) .
\end{aligned}
$$

As shown in equation (27), the expression of antiresonance frequency contains the information of the location of the driving point. Therefore, the sensitivity of the antiresonance frequency $z_{j}$ with respect to the location of driving point $j$ can be derived as follows:

$$
\begin{aligned}
& \frac{\partial z_{j}}{\partial x_{j}}=-\sqrt{\frac{3 G}{\rho}} \frac{3 \pi k_{1}(1+\theta)}{x_{j}^{2}\left(\cos \left(\pi k_{1} l / x_{j}\right)+2+3 \theta\right)^{3 / 2}} \cos \frac{\pi k_{1} l}{2 x_{j}}\left(k_{1}=1,2, \ldots, j-1\right), \\
& \frac{\partial z_{j}}{\partial x_{j}}=\sqrt{\frac{3 G}{\rho}} \frac{3 \pi\left(2 k_{2}-1\right)(1+\theta)}{4\left(N_{P} l-x_{j}\right)^{2}\left[\cos \left(\pi\left(2 k_{2}-1\right) l / 2\left(N_{P} l-x_{j}\right)\right)+2+3 \theta\right]^{3 / 2}} \cos \frac{\pi\left(2 k_{2}-1\right) l}{4\left(N_{P} l-x_{j}\right)}\left(k_{2}=1,2, \ldots, N_{P}-j\right) .
\end{aligned}
$$

The antiresonance frequency with the larger sensitivity coefficient $\partial z_{j} / \partial x_{j}$ is expected to be much more sensitive to the location of driving point $j$. However, the sensitivity coefficient $\partial z_{j} / \partial x_{j}$ is closely related to the order of antiresonance frequency $z_{j}$, so the antiresonance frequencies of different orders cannot be directly fused together for the selection of the optimal driving point and damage identification. Therefore, the relative sensitivity $\partial \bar{z}_{j} / \partial x_{j}$ is proposed, defined by

$$
\frac{\partial \bar{z}_{j}}{\partial x_{j}}=\frac{1}{z_{j}} \frac{\partial z_{j}}{\partial x_{j}},
$$

$\partial \bar{z}_{j} / \partial x_{j}$ represents the sensitivity of the change rate of antiresonance frequency to the location of driving point, which can be used to eliminate the influence of the different orders.

In the evaluation of the $j$-th node contributions in the sensitivities of the antiresonances, the sensitivity coefficient $\partial \bar{z}_{j} / \partial x_{j}$ is normalised.

$$
c_{j, n}=\frac{\left|\partial \bar{z}_{j, n} / \partial x_{j}\right|}{\sum_{j=1}^{N_{P}}\left|\partial \bar{z}_{j, n} / \partial x_{j}\right|} .
$$

Assuming that only the first $N_{m}$ order antiresonance frequencies of each driving point are available, the vector containing the sensitivity coefficients $c_{j, n}$ can be formed as $C_{j}=\left[c_{j, 1}, c_{j, 2}, \ldots, c_{j, N_{m}}\right]$. The overall sensitivity of antiresonance frequency of point $j$ can be expressed as

$$
\bar{C}_{j}=\sum_{n=1}^{N_{m}} c_{j, n} .
$$

With the index $\bar{C}_{j}$, the antiresonance frequency sensitivities involving the much higher value are expected to be optimal for damage identification.

\section{Sensitivity-Based Damage Detection}

When the ideal periodicity is disrupted due to the damage, the laminated rubber bearing becomes a quasiperiodic structure. The changes in the $n$-th antiresonance frequency of the $j$-th node before and after the damage can be expressed as

$$
\Delta z_{j, n}=z_{j, n}^{d}-z_{j, n}^{u}
$$

where $z_{j, n}^{d}$ and $z_{j, n}^{u}$ are the $n$-th antiresonance frequencies of the $j$-th node in the damaged and undamaged statuses, respectively.

The damage of the shear modulus is considered in this study. Take as an example the case in which the driving point is at the $j$-th node.

$$
\left\{\begin{array}{c}
\frac{\partial z_{j, 1}}{\partial G^{[1]}} \Delta G^{[1]}+\frac{\partial z_{j, 1}}{\partial G^{[2]}} \Delta G^{[2]}+\cdots+\frac{\partial z_{j, 1}}{\partial G^{\left[N_{P}\right]}} \Delta G^{\left[N_{P}\right]} \cong \Delta z_{j, 1} \\
\frac{\partial z_{j, 2}}{\partial G^{[1]}} \Delta G^{[1]}+\frac{\partial z_{j, 2}}{\partial G^{[2]}} \Delta G^{[2]}+\cdots+\frac{\partial z_{j, 2}}{\partial G^{\left[N_{P}\right]}} \Delta G^{\left[N_{P}\right]} \cong \Delta z_{j, 2} \\
\vdots \\
\frac{\partial z_{j, N_{m}}}{\partial G^{[1]}} \Delta G^{[1]}+\frac{\partial z_{j, N_{m}}}{\partial G^{[2]}} \Delta G^{[2]}+\cdots+\frac{\partial z_{j, N_{m}}}{\partial G^{\left[N_{P}\right]}} \Delta G^{\left[N_{P}\right]} \cong \Delta z_{j, N_{m}}
\end{array}\right.
$$

Suppose the number of driving points selected is $N_{D}$. Thus, the total number of available changes in the antiresonance frequency is $q=N_{D} \times N_{m}$. Equation (33) is applied to all driving points, and a matrix form is written as

$$
\mathbf{S} \Delta \mathbf{G}=\Delta \mathbf{z},
$$


where $S \in R^{q \times N_{P}}$ is the sensitivity matrix of the antiresonance frequencies with respect to the shear modulus. Furthermore, $\Delta \mathbf{z} \in \mathrm{R}^{q \times 1}$ is the vector consisting of the change in measured antiresonance frequencies, and $\Delta \mathbf{G}=$ $\left[\Delta G_{1}, \Delta G_{2}, \ldots, \Delta G_{N_{P}}\right]^{T} \in \mathrm{R}^{N_{P} \times 1}$ is the parameter vectors to be identified.

In practical engineering structures, the damage types of the isolation bearings are varied. As the ageing of rubber material leads to the increase in the shear modulus, the inequality constraint $\Delta G \geq 0$ is introduced. The optimality criterion used in $[17,18]$ is adopted to solve equation (34).

Thus, by solving the nonnegative least-square curve fitting problem [36], the optimal solution of the change in shear modulus of each cell can be obtained.

$$
\min _{\Delta G \geq 0} \frac{1}{2}\|\mathbf{S} \Delta \mathbf{G}-\Delta \mathbf{z}\|^{2} .
$$

It should be pointed out that the damage usually only occurs in a small part of the entire structure, which means the damage vector $\Delta G$ is sparse. It guarantees the uniqueness of the solutions to underdetermined linear systems of equations (37).

\section{Analytical Examples}

A rubber-steel periodic structure with one fixed end and one free end is employed to demonstrate the feasibility of the proposed method in the damage identification of a laminated rubber bearing. As shown in Figure 1, the rubber-steel quasiperiodic structure consists of $N_{P}$ cells, in which the cells are numbered starting at 1 from the bottom cell to the top one. The structural parameters are as follows: the thickness of each rubber layer is $l=0.01 \mathrm{~m}$, the cross-sectional diameter is $\varphi=0.8 \mathrm{~m}$, the shear modulus is $G=1 \times 10^{6} \mathrm{~N} / \mathrm{m}^{2}$, the density is $\rho=1000 \mathrm{~kg} / \mathrm{m}^{3}$, the mass ratio of steel to rubber is $\theta=2$, and the total number of cells is $N_{P}=10$. The details are listed in Table 1.

The method is tested for its ability to detect damage by using five cases. The detailed damage configuration is shown in Table 2. The cases simulate the increase in shear modulus of the rubber layer due to the ageing of the rubber material. Cases 1 and Case 2 are single damage conditions in which the corresponding shear moduli of cells 1 and 10 are increased by $5 \%$. Case 3 has two damaged cells, which shows the condition in which the shear moduli of cells 1 and 10 are increased by $5 \%$ simultaneously. Cases 4 and 5 have three damaged cells. Case 4 shows the condition in which the shear moduli of cells 2,7 , and 10 are increased by $8 \%, 2 \%$, and $5 \%$, respectively. In Case 5, the damage appears in cells 2,4 , and 6 , and the damage magnitudes of the three cells are all increased by $5 \%$.

The optimal driving points are selected according to the selection criteria in accordance with the more sensitive driving points proposed in Section 3.2. The specific process is as follows. Firstly, by substituting the geometric and material parameters into equation (19), the $n$-th antiresonance frequencies of the $j$-th node in the healthy status, which is given by $z_{j, n}^{u}\left(j=1,2, \ldots, N_{P} ; n=1,2, \ldots, N_{m}\right)$, can be calculated. Assume that only the first four antiresonance frequencies of each driving point are used similar to that in a real situation in which only limited measurement information is available, i.e., $N_{m}=4$. The first four antiresonance frequencies of the different driving points are listed in Table 3. Secondly, the sensitivity coefficients of the antiresonance frequencies with respect to the location of driving points are calculated using equation. (28), as depicted in Figure 3(a). Finally, the overall sensitivity of the antiresonance frequencies of driving point $j$ is obtained using equation (31). As shown in Figure 3(c), the three most sensitive points are the 6th, 5th, and 7th nodes, whereas the two least sensitive points are the 9th and 10th node.

From the calculation of the overall sensitivity of the driving points, five schemes are formulated for driving point selection in damage identification, as shown in Table 4. In Schemes 1 and 2, the 6th and 5th nodes are selected as the driving points, respectively. In Scheme 3 , the 6th and 5th nodes are simultaneously selected as the driving points. In Scheme 4, the 6th, 5th, and 7th nodes are selected as the driving points to investigate the influence of the size of the "optimal driving point" dataset on the damage identification. Scheme 5, which consists of the "worst two" driving points (9th and 10th nodes), is also investigated.

Take Case 1 as an example for demonstrating the process of calculation when the 1 st node is used as the only driving point, i.e., $j=6$ and $N_{D}=1$. And the procedure of damage detection is illustrated in Figure 4. Firstly, by substituting the geometric and material parameters into equation (25), the sensitivity coefficients of antiresonance frequencies with respect to the shear modulus are calculated. As shown in Figure 5, the sensitivity coefficients of the element in the substructure on one side of the driving point are equal to zero. The sensitivity of the driving point antiresonance frequency represents the sensitivity of the natural frequency of the substructure on either side of the driving point. Consequently, the sensitivity of the antiresonance frequency to the element in another substructure equals zero.

Secondly, by substituting the geometric and material parameters for Case 1 (i.e., $\Delta G^{[1]}=5 \% G$ ) into equation (8), the antiresonance frequencies in the damaged status $z_{j, n}^{d}$ are calculated. Then, the changes in antiresonance frequencies before and after the damage are computed according to equation (32). Finally, by using the change in antiresonance frequencies and the sensitivity matrix, the damage is identified by solving the optimisation problem of equation (35). The results of the damage identification are expressed using the change rate of the shear modulus, which is defined as $\Delta G / G$. Subsequently, Case 1 is detected using the other four schemes of the driving point selection. As shown in Figure 6 , when only the 5 th or 6 th node is used, the damage in cell 1 is misidentified due to the symmetry. In other words, the sensitivity of antiresonance frequency of the 5th node to cell 1 is equal to that of cell 5 , and the sensitivity of antiresonance frequency of the 6th node to cell 1 is equal to that of cell 6, as shown in Figure 5(a) and 5(b). These findings indicate that the antiresonance frequencies of a single driving point cannot be used for damage identification even if it is the optimal driving point. The results of damage identification, which uses the "best two" and "best three" 
TABLE 1: The geometric and material parameters of periodic rubber-steel structure.

\begin{tabular}{lc}
\hline Parameters & Values \\
\hline Static shear modulus $(G)$ & $1 \times 10^{6} \mathrm{~N} / \mathrm{m}^{2}$ \\
Cross section diameter of the rubber layer $(\varphi)$ & $0.8 \mathrm{~m}$ \\
Density of the rubber material $(\rho)$ & $1000 \mathrm{~kg} / \mathrm{m}^{3}$ \\
Thickness of each rubber layer $(l)$ & $0.01 \mathrm{~m}$ \\
Mass ratio of steel to rubber $(\theta)$ & 2 \\
The total number of cells $\left(N_{P}\right)$ & 10 \\
\hline
\end{tabular}

TABLe 2: Damage cases for the 10-cell periodic rubber-steel structure.

\begin{tabular}{lccccc}
\hline Cases & Case 1 & Case 2 & Case 3 & Case 4 & Case 5 \\
\hline Damage cell $(k)$ & 1 & 10 & 1,10 & $2,7,10$ & $2,4,6$ \\
Increase in shear modulus $(\Delta G / G) / \%$ & 5 & 5 & 5,5 & $8,2,5$ & $5,5,5$ \\
\hline
\end{tabular}

TABLE 3: The first four antiresonance frequencies of different driving points $(j=1,2, \ldots, 10)$ for undamaged 10 -cell periodic rubber-steel structure.

\begin{tabular}{|c|c|c|c|c|c|c|c|c|c|c|}
\hline Order $(n)$ & $z_{1, n}^{u}$ & $z_{2, n}^{u}$ & $z_{3, n}^{u}$ & $z_{4, n}^{u}$ & $z_{5, n}^{u}$ & $z_{6, n}^{u}$ & $z_{7, n}^{u}$ & $z_{8, n}^{u}$ & $z_{9, n}^{u}$ & $z_{10, n}^{u}$ \\
\hline 1 & 318.5167 & 358.2907 & 409.4071 & 477.5191 & 572.7775 & 715.4009 & 817.0391 & 715.4009 & 636.2086 & 572.7775 \\
\hline 2 & 952.1873 & 1070.0356 & 1220.8973 & 2320.4851 & 1140.5367 & 952.1873 & 952.1873 & 2102.0259 & 1265.4368 & 1140.5367 \\
\hline 3 & 1574.7513 & 1765.4390 & 1878.6729 & 2738.6128 & 1697.0584 & 1878.6729 & 1618.5364 & 2738.6128 & 1878.6729 & 1697.0584 \\
\hline 4 & 2175.4360 & 2427.5732 & 2006.8487 & 3123.5874 & 2233.7496 & 2102.0259 & 2381.8669 & 3300.1676 & 2462.9191 & 2233.7496 \\
\hline
\end{tabular}

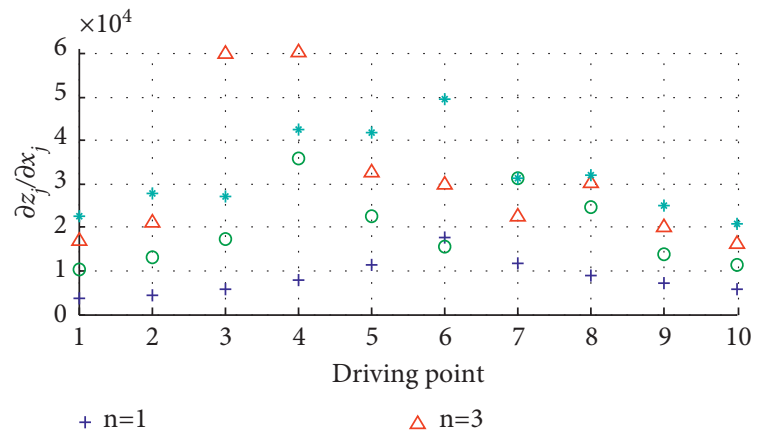

$\mathrm{n}=2$
* $\mathrm{n}=4$

(a)
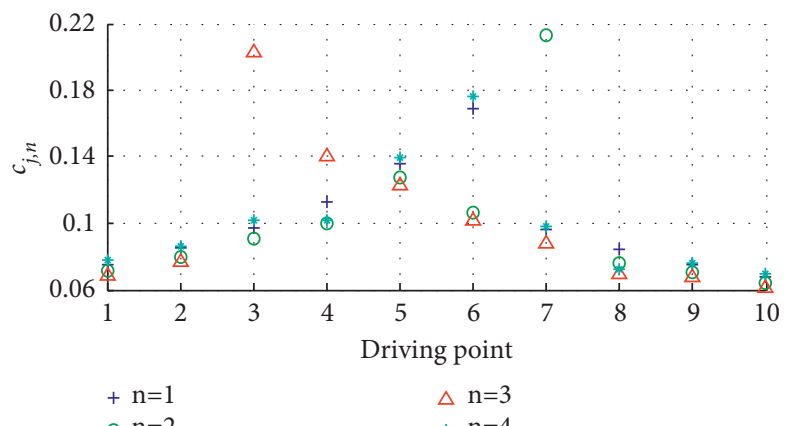

(b)

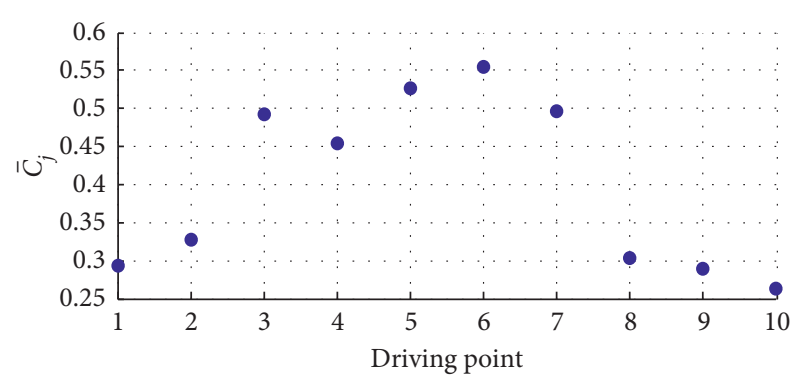

(c)

Figure 3: (a) The sensitivity coefficients $\partial z_{j, n} / \partial x_{j}\left(j=1,2, \ldots, N_{P} ; n=1,2, \ldots, N_{m}\right)$ for $N_{m}=4$ and $N_{P}=10$. (b) The normalised sensitivity coefficients $c_{j, n}\left(j=1,2, \ldots, N_{P} ; n=1,2, \ldots, N_{m}\right)$. (c) The overall sensitivity $\bar{C}_{j}$ of driving point $j\left(j=1,2, \ldots, N_{P}\right)$.

driving points, suggest that the location and magnitude of the damage can be both predicted reasonably. Therefore, more than one driving point should be selected for damage identification.
From the aforementioned analyses, Schemes 3 and 4 are selected for damage identification. Scheme 5, which consists of the "worst two" driving points, is also studied to verify the proposed optimal driving point selection criteria. In 


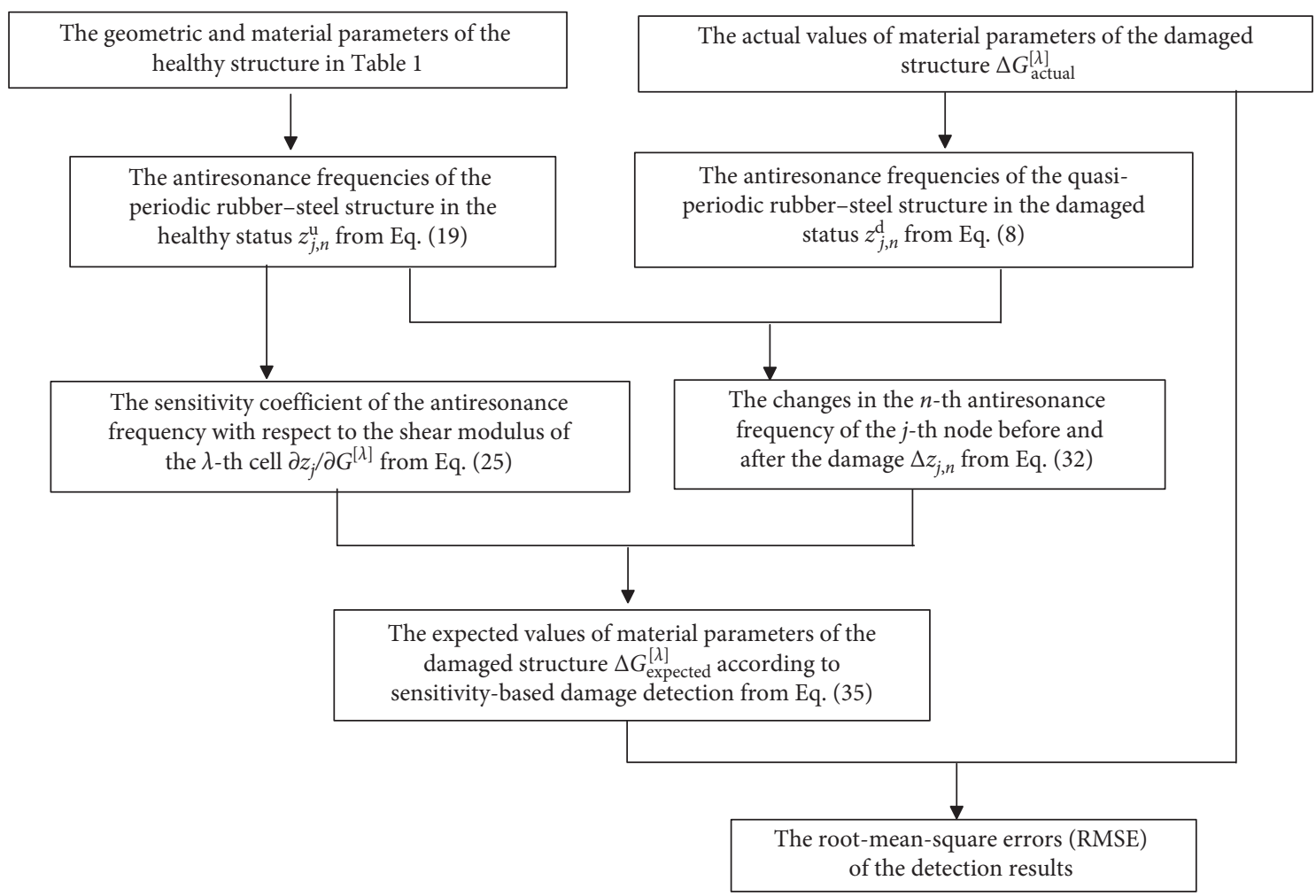

FIgURE 4: The procedure of damage detection in laminated rubber bearing.

TABLE 4: Selection of driving points.

\begin{tabular}{lccccc}
\hline Schemes & $(1)$ & $(2)$ & $(3)$ & $(4)$ & $(5)$ \\
\hline Driving points & 6 & 5 & 5,6 & $5,6,7$ & 9,10 \\
\hline
\end{tabular}

addition, the damage detection result of the proposed method that uses antiresonance frequencies is compared with that of the scheme that uses natural frequencies (the first five orders). In this study, only the first five natural frequencies are used to simulate the situation in which only the fewer-order modes can be measured in practical application [17]. The accuracy of the identification results is measured using the root mean square error (RMSE), which is normalised by the initial parameter value, $\operatorname{RMSE}=\sqrt{1 / N_{P} \sum_{\lambda=1}^{N_{P}}\left(\Delta G_{\text {exp ected }}^{[\lambda]}-\Delta G_{\text {actual }}^{[\lambda]} / G\right)^{2}}\left(\lambda=1,2, \ldots, N_{P}\right)$ [29]. Table 5 shows the RMSE of the identification results of each damage identification scheme under different damage conditions. The process is repeated to detect a single damage in the 10th cell. As shown in Figures 7(1) and 7(2), both of the antiresonance frequencies extracted from more than one driving point and natural frequencies offer good predictions of the single damage, with the RMSE approaching zero. The small deterioration in terms of accuracy of the damage magnitude prediction can be attributed to the sensitivity coefficients being deduced around the healthy structure.

Then, a detection of the two damaged cells is conducted on the basis of the abovementioned two single damage cases. The detection result of Case 3 is shown in Figure 7(3). The damage in cells 1 and 10 can be identified using the antiresonance frequencies of more than one driving point. When natural frequencies are used, the damage in cell 10 cannot be identified, and the cell is even incorrectly identified as cell 9. The corresponding RMSE approaches a value as high as $1.7 \%$.

Subsequently, the three cells in the structure are utilised for damage detection. In Case 4, the damage types in cells 2,7 , and 10 can be identified using the antiresonance frequencies of more than one driving point. When natural frequencies are used, the damage in cell 10 cannot be identified, and the cell is even incorrectly identified as cell 1, as shown in Figure 7(4). In Case 5, the damage types in cells 2,4 , and 6 can be predicted reasonably using either the antiresonance frequencies of more than one optimal driving point or the natural frequencies. When the "worst two" driving points are used, the damaged cells cannot be identified. The RMSE of the damage identification results entailing the "worst two" driving points selected by the proposed criterion is much greater than that involving the "best two" driving points, as shown in Table 5. On the one hand, as shown in Figure 8, the performance of the antiresonance frequencies of the "best two" driving points selected by the proposed criterion is better than that of the natural 

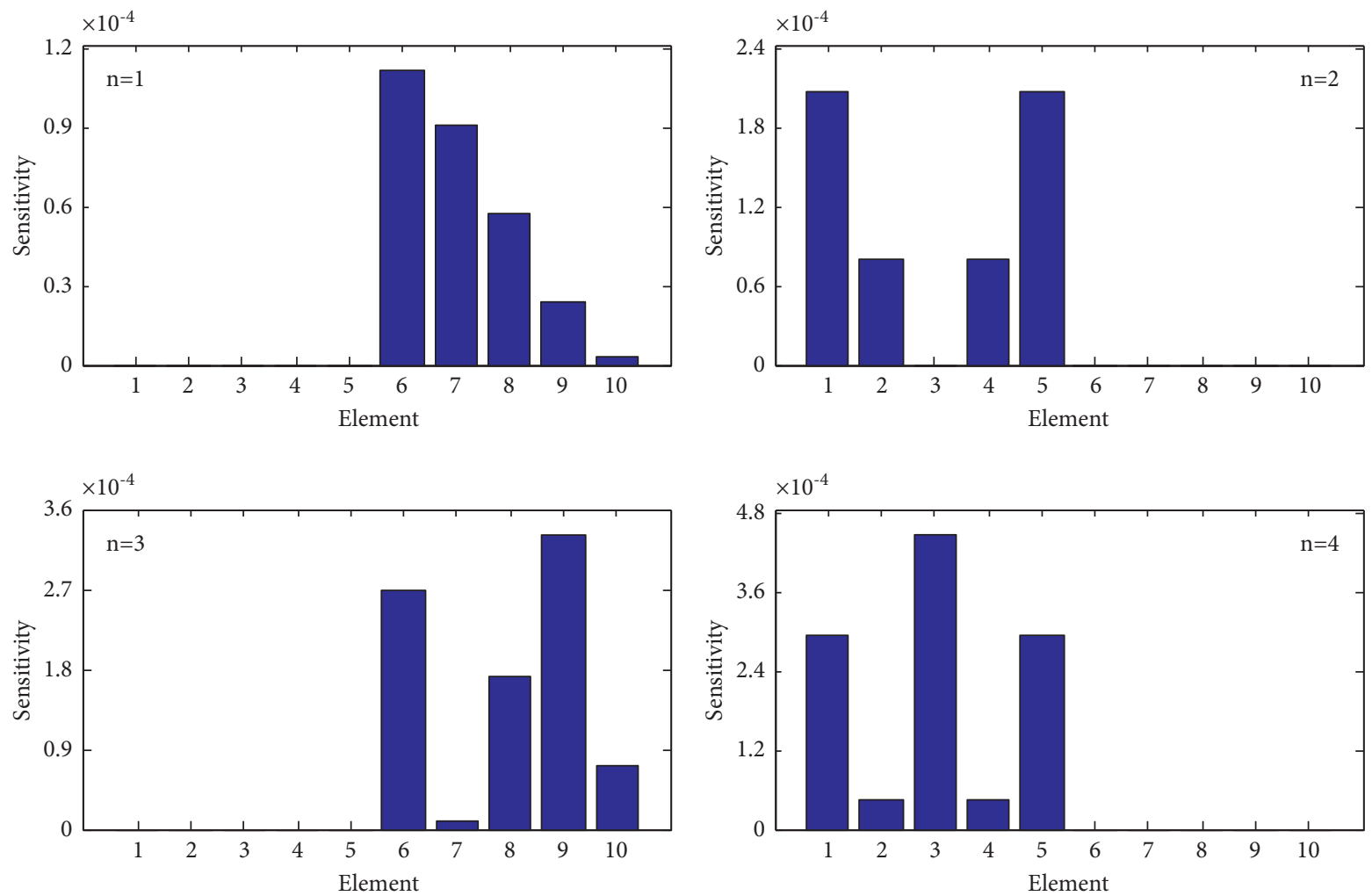

(a)
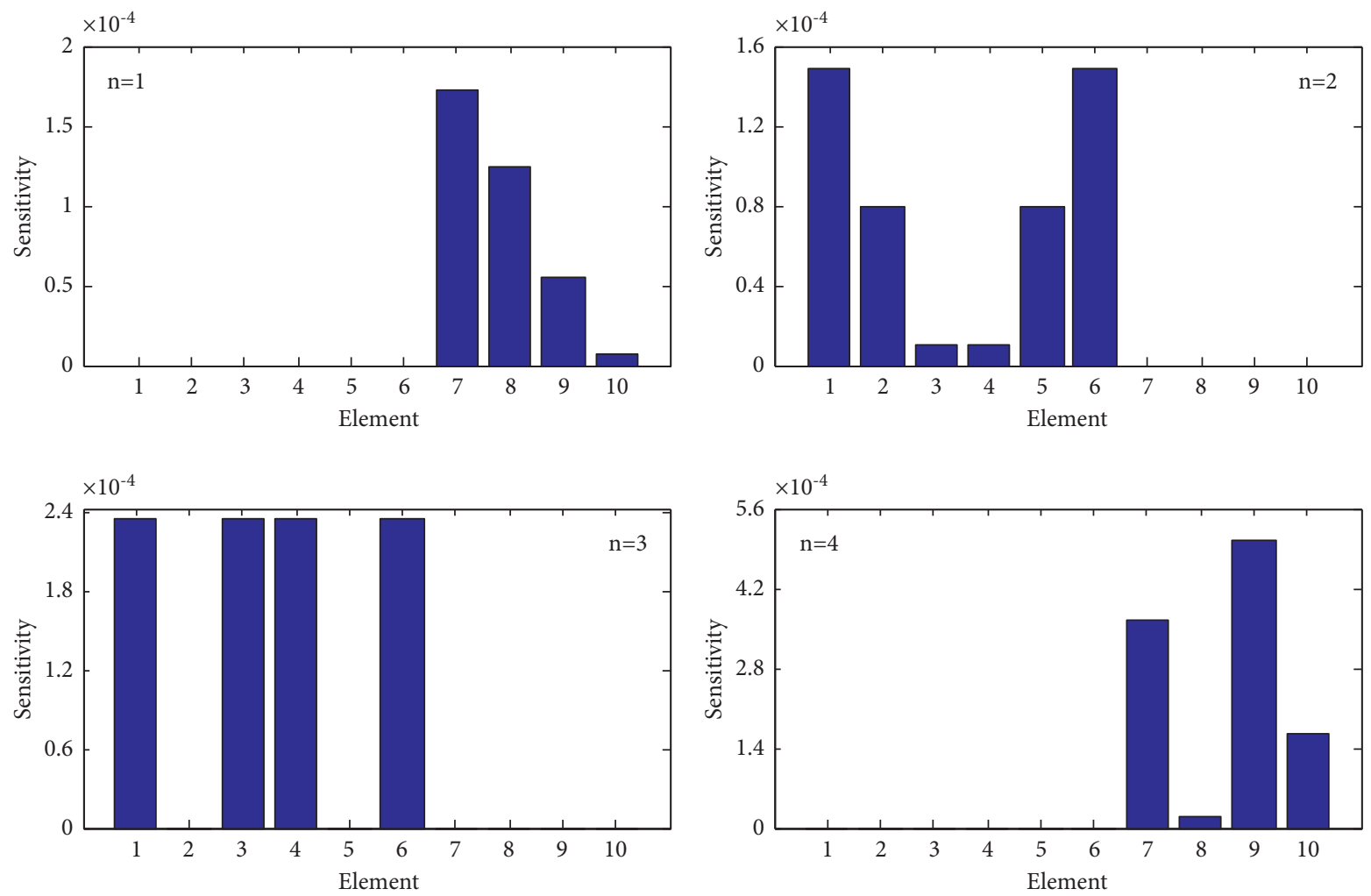

(b)

FIgURE 5: Continued. 

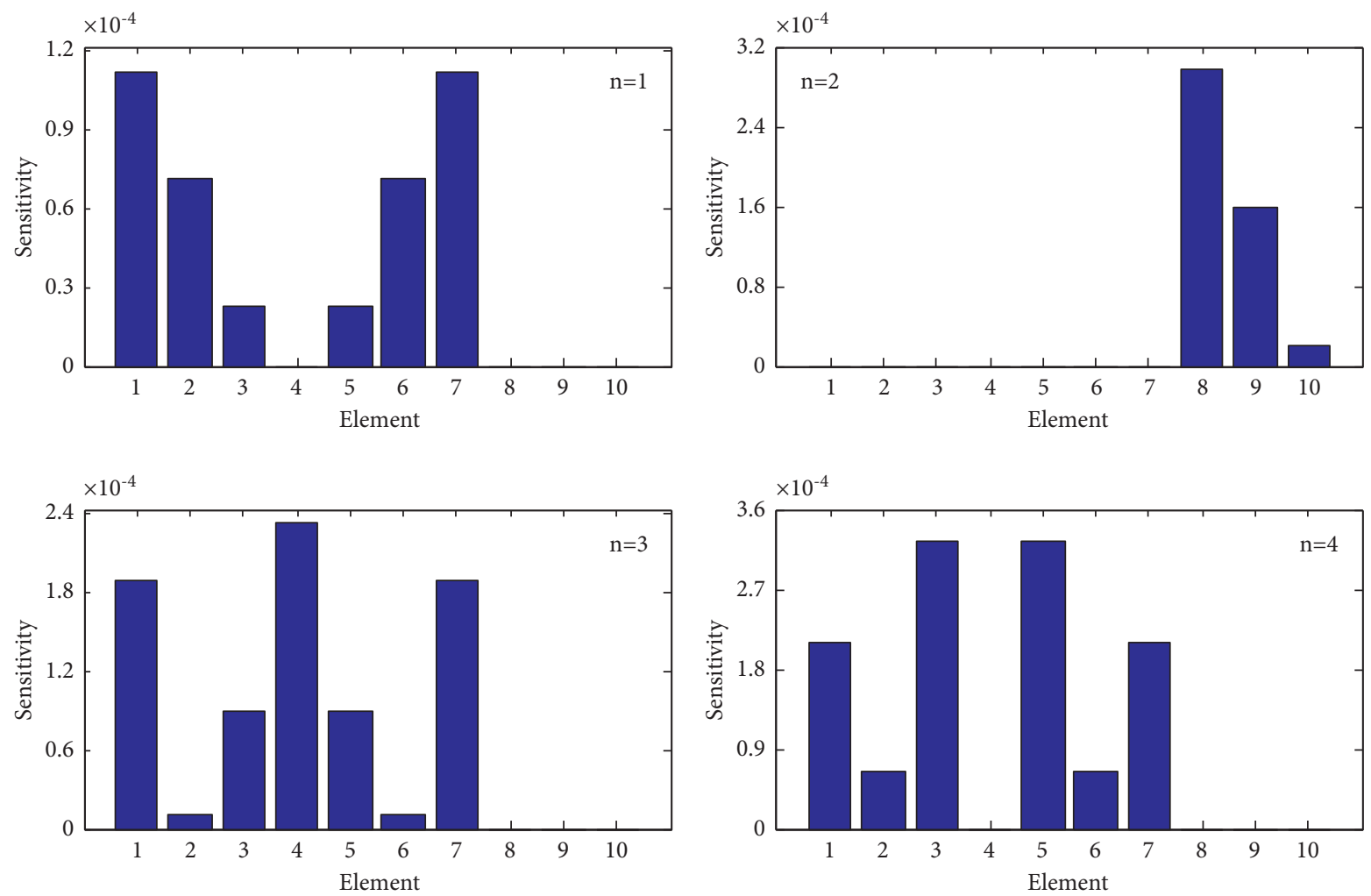

(c)
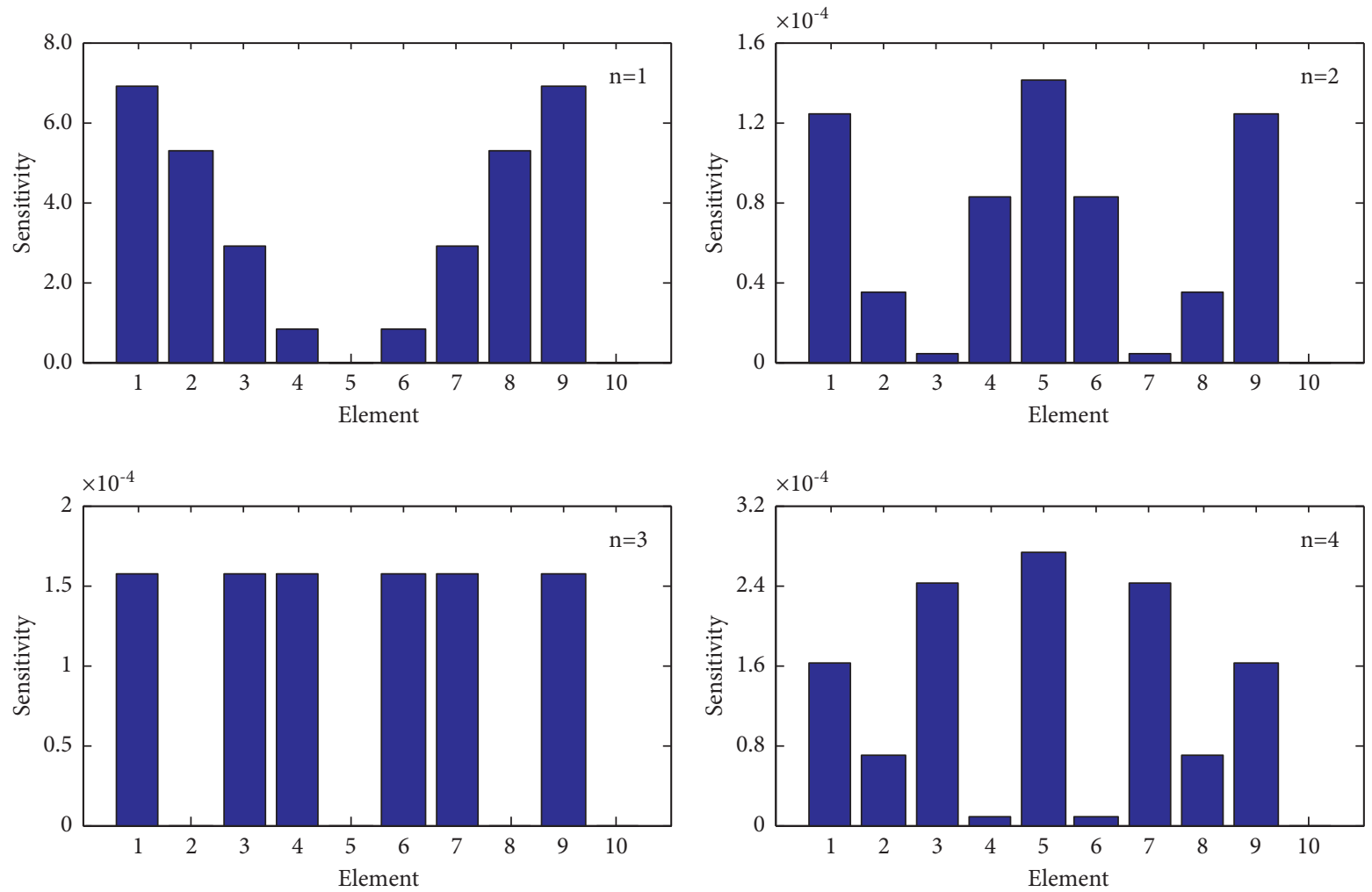

(d)

Figure 5: Continued. 

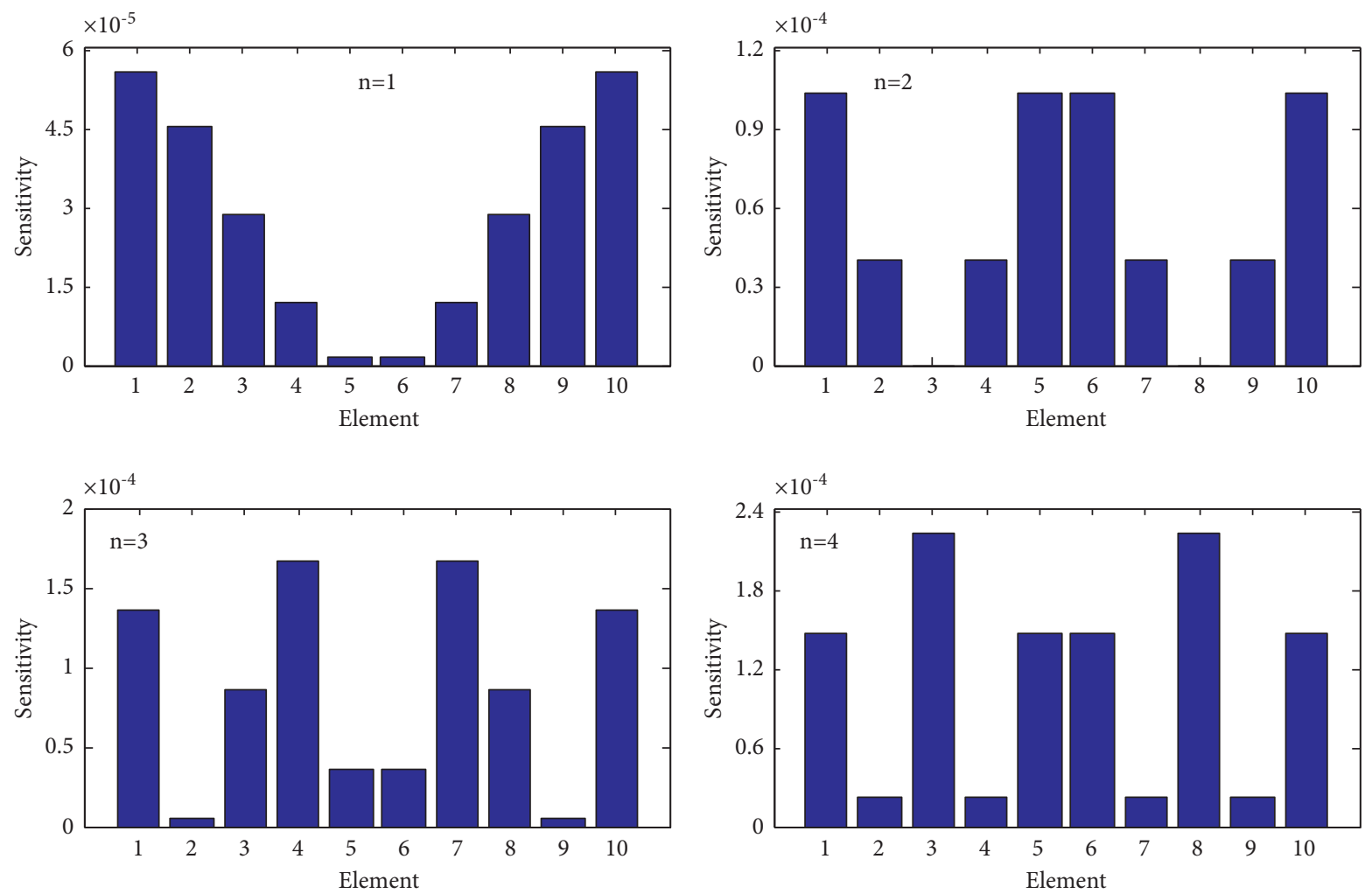

(e)

Figure 5: The sensitivity coefficients $\partial z_{j, n} / \partial G^{[\lambda]}\left(j=1,2, \ldots, N_{P} ; n=1,2, \ldots, N_{m} ; \lambda=1,2, \ldots, N_{P}\right)$. (a) $j=5$. (b) $j=6$. (c) $j=7$. (d) $j=9$. (e) $j=10$.

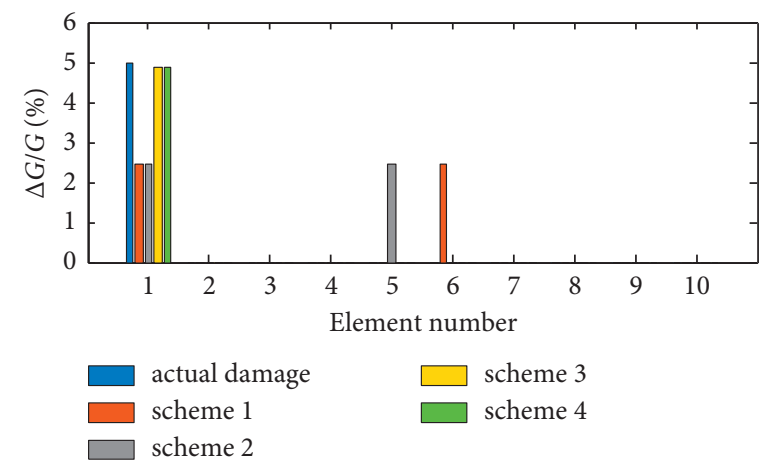

Figure 6: Detection of Case 1 using Schemes 1-4.

TABLE 5: RMSE (\%) of the 5 cases.

\begin{tabular}{llllll}
\hline & Case 1 & Case 2 & Case 3 & Case 4 & Case 5 \\
\hline Scheme 3 & 0.0425 & 0.1060 & 0.1199 & 0.1674 & 0.1126 \\
Scheme 4 & 0.0431 & 0.1079 & 0.1228 & 0.1664 & 0.1082 \\
Scheme 5 & 0.0482 & 0.0495 & 0.0638 & 0.1845 & 2.2470 \\
Natural frequencies & 0.0508 & 0.1226 & 1.6846 & 1.7771 & 0.1114 \\
\hline
\end{tabular}

frequencies. On the other hand, the performance of the antiresonance frequencies of the "best three" driving points selected by the proposed criterion is less superior to that of the "best two" driving points. The analytic results demonstrate that the "best two" driving points can provide sufficient information for identification. 


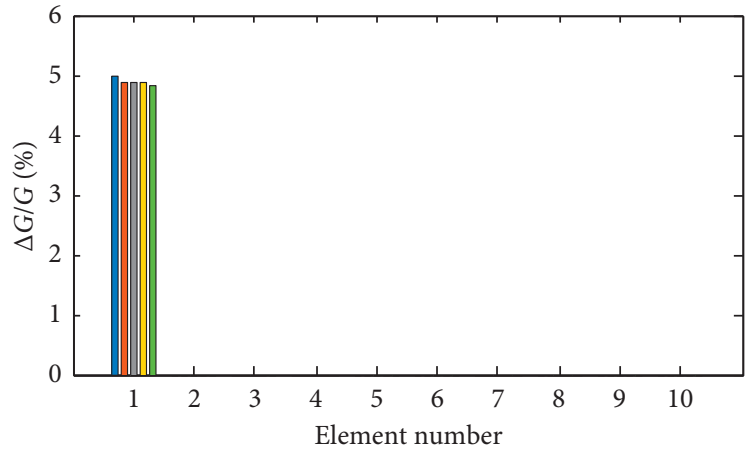

(a)

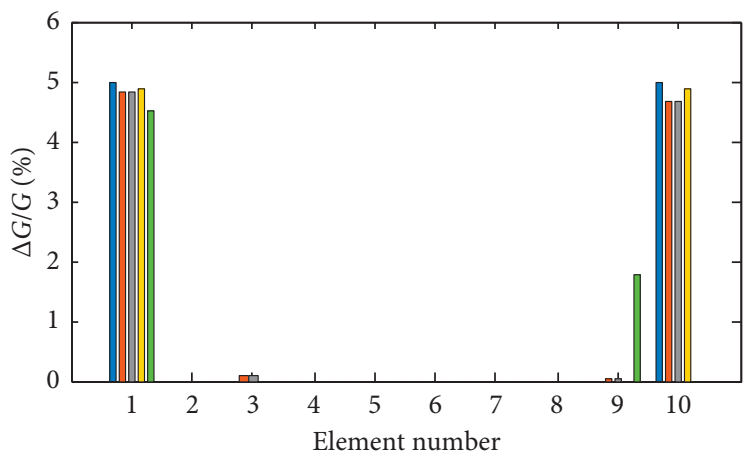

(c)

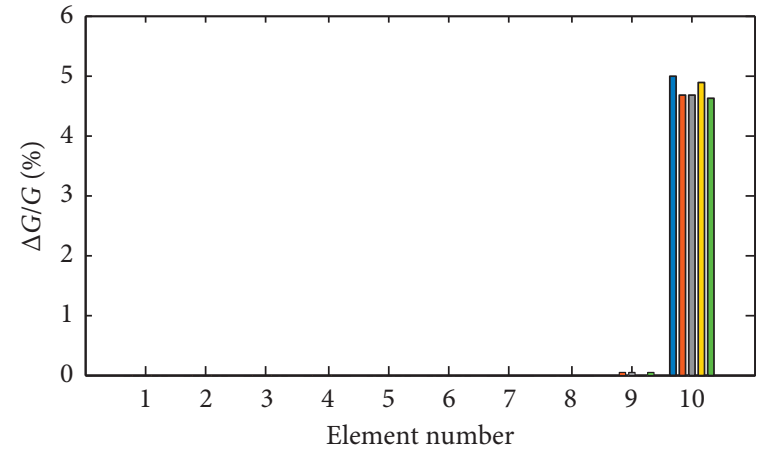

(b)

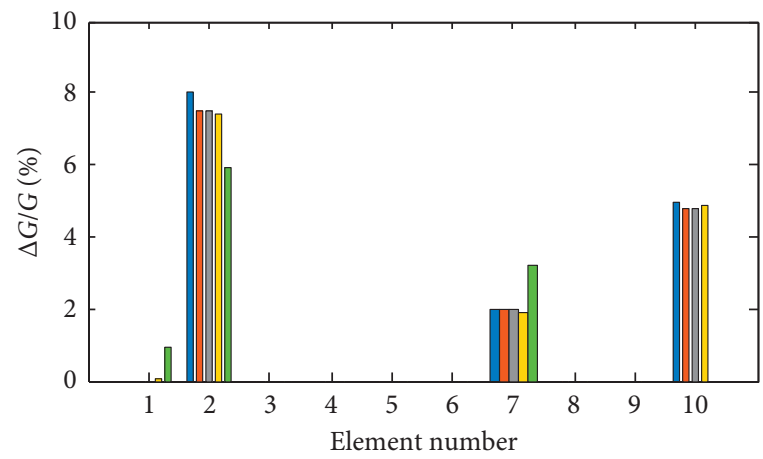

(d)

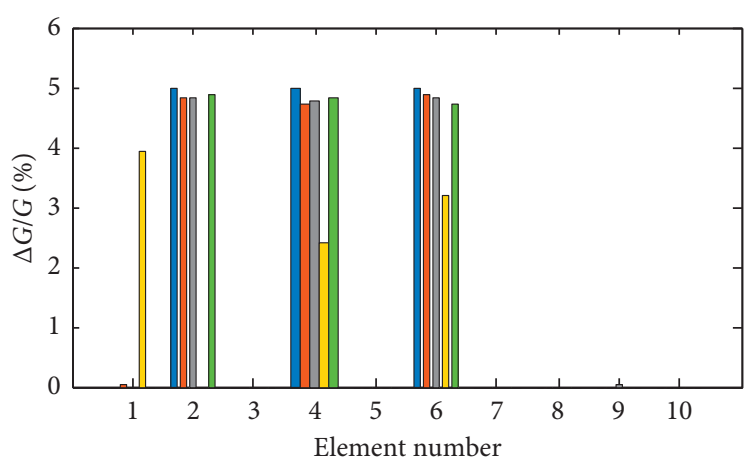

(e)

Figure 7: Detection of Cases 1-5. $\approx$ Actual damage; $\approx$ identification result (Scheme 3); $\approx$ identification result (Scheme 4); $₫$ identification result (Scheme 5); - identification result (natural frequencies).

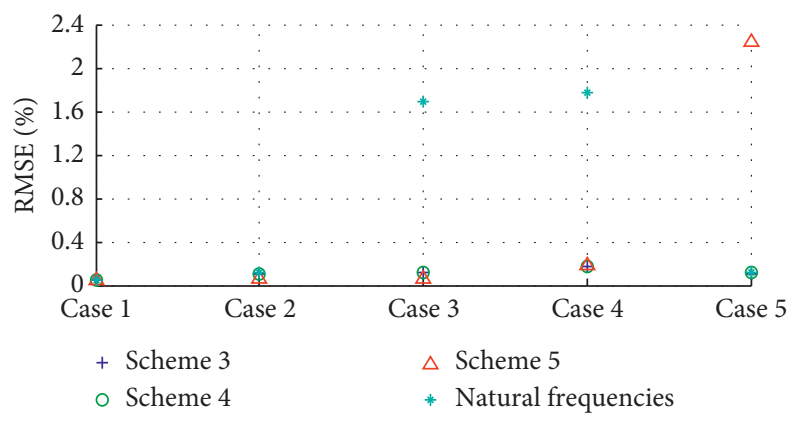

Figure 8: Root mean square errors (RMSE) of the detection results for different cases. 


\section{Conclusions}

This study presented an effective method of damage detection for rubber bearings based on the antiresonance frequencies of a periodic structure and its sensitivity analysis. The laminated rubber bearing was simplified as a monocoupled rubber-steel periodic structure. The antiresonance frequency of the monocoupled periodic structure was derived using the dynamic stiffness method combined with the periodicity of the structure. The optimal driving points were selected on the basis of the sensitivity analysis of the antiresonance frequency on the location of the driving point. The sensitivity of the antiresonance frequency to the damage scaling parameter was derived from the antiresonance frequency characteristic equation. The expressions of antiresonance frequency as well as its sensitivities are explicit, which have application to engineers and designers. Then, the changes in antiresonance frequencies were measured. The damage was identified by solving the sensitivity identification equation by means of the numerical optimisation method.

The numerical case studies were performed under different damage scenarios. The identification results showed that the antiresonance frequencies can be used to identify the shear modulus damage of different rubber layers, and the identification result entailing antiresonance frequencies is better than that involving natural frequencies. The driving point antiresonance frequencies selected by the optimal driving points selection criterion can always produce better identification results. Moreover, this study demonstrated that the "best two" driving points can provide sufficient information for damage identification. It should be emphasized that compared with the most of laminated rubber bearings used in the practical engineering structures, the periodic system model considered in this paper is relatively simple. For example, the bending deformation of the rubber layer is neglected. Therefore, the performance of the approach for multicoupled periodic systems with complex boundary conditions needs to be investigated. Besides, there exists another matter of how to consider dampness and nonlinearity of rubber materials.

\section{Appendix}

The detailed expressions of characteristic determinants of the substructures on both sides of the driving point, i.e., $\left|\mathbf{D}_{\mathrm{L}}\right|$ and $\left|\mathbf{D}_{R}\right|$, are as follows:

$$
\begin{aligned}
& \left|\mathbf{D}_{L}\right|=\left|\begin{array}{ccccc}
d_{R R}^{[1]}+d_{L L}^{[2]} & d_{L R}^{[2]} & & & \\
d_{R L}^{[2]} & d_{R R}^{[2]}+d_{L L}^{[3]} & d_{L R}^{[3]} & & \\
& \ddots & \ddots & \ddots & \\
& & d_{R L}^{[j-2]} & d_{R R}^{[j-2]}+d_{L L}^{[j-2]} & d_{L R}^{[j-2]} \\
& & & d_{R L}^{[j-1]} & d_{R R}^{[j-1]}+d_{L L}^{[j]}
\end{array}\right|_{(j-1) \times(j-1)} \\
& \left|\mathbf{D}_{\mathrm{R}}\right|=\left|\begin{array}{ccccc}
d_{R R}^{[j+1]}+d_{L L}^{[j+2]} & d_{L R}^{[j+2]} & & & \\
d_{R L}^{[j+2]} & d_{R R}^{[j+2]}+d_{L L}^{[j+3]} & d_{L R}^{[j+3]} & & \\
& \ddots & \ddots & \ddots & \\
& & & d_{R R}^{\left[N_{P}-1\right]}+d_{L L}^{\left[N_{P}\right]} & d_{L R}^{\left[N_{P}\right]} \\
& & & d_{R L}^{\left[N_{P}\right]} & d_{R R}^{\left[N_{P}\right]}
\end{array}\right|_{\left(N_{P}-j\right) \times\left(N_{P}-j\right)}
\end{aligned}
$$

The determinant $\left|\bar{D}_{\mathrm{L}}\right|$ can be calculated by expanding the $\lambda-$ th $(\lambda=1,2, \ldots, j)$ row.

$$
\left|\overline{\mathbf{D}}_{L}\right|=\frac{d_{R L}^{[\lambda]}}{d_{L R}}\left|\overline{\mathbf{D}}_{L}\right|_{\lambda, \lambda-1}+\left(\cosh \mu-\frac{d_{L L}^{[\lambda]}}{d_{L R}}\right)\left|\overline{\mathbf{D}}_{L}\right|_{\lambda, \lambda}+\left|\overline{\mathbf{D}}_{L}\right|_{\lambda, \lambda+1} .
$$

The determinant $\left|\bar{D}_{\mathrm{L}}\right|_{\lambda, \lambda-1}$ is obtained by removing the $\lambda$ - th row and the $(\lambda-1)$ - th column of $\left|\bar{D}_{\mathrm{L}}\right|$; the determinant $\left|\bar{D}_{\mathrm{L}}\right|_{\lambda, \lambda}$ is obtained by removing the $\lambda$ - th row and the $\lambda$ - th column; and the determinant $\left|\bar{D}_{\mathrm{L}}\right|_{\lambda, \lambda+1}$ is obtained by removing the $\lambda-$ th row and the $(\lambda+1)-$ th column. The detailed expressions of the determinants $\left|\bar{D}_{\mathrm{L}}\right|_{\lambda, \lambda-1},\left|\bar{D}_{\mathrm{L}}\right|_{\lambda, \lambda}$, and $\left|\bar{D}_{\mathrm{L}}\right|_{\lambda, \lambda+1}$ are as follows: 


$$
\begin{aligned}
\left|\overline{\mathbf{D}}_{L}\right|_{\lambda, \lambda-1} & =-\frac{d_{L R}^{[\lambda]}}{d_{L R}} \frac{\sinh (\lambda-1) \mu \sinh (j-\lambda) \mu}{\sinh h^{2} \mu}, \\
\left|\overline{\mathbf{D}}_{L}\right|_{\lambda, \lambda} & =\frac{\sinh (j-\lambda) \mu}{\sin h \mu}\left[\left(\cosh \mu-\frac{d_{L L}^{[\lambda]}}{d_{L R}}\right) \frac{\sinh ((\lambda-1)) \mu}{\sinh \mu}-\frac{\sinh ((\lambda-2)) \mu}{\sinh \mu}\right], \\
\left|\overline{\mathbf{D}}_{L}\right|_{\lambda, \lambda+1} & =-\frac{\sinh (j-\lambda-1) \mu}{\sinh \mu}\left[\left(\cosh \mu-\frac{d_{L L}^{[\lambda]}}{d_{L R}}\right) \frac{\sinh (\lambda-1) \mu}{\sinh \mu}-\frac{\sinh (\lambda-2) \mu}{\sinh \mu}\right] .
\end{aligned}
$$

The determinant $\left|\bar{D}_{\mathrm{R}}\right|$ can be calculated by expanding the $(\lambda-j)-$ th $\left(\lambda=j+1, j+2, \ldots, N_{P}\right)$ row.

$$
\begin{aligned}
\left|\overline{\mathbf{D}}_{\mathrm{R}}\right|= & \frac{d_{L R}^{[\lambda]}}{d_{L R}}\left|\overline{\mathbf{D}}_{\mathrm{R}}\right|_{\lambda-j, \lambda-j-1} \\
& +\left(\cos h \mu-\frac{d_{L L}^{[\lambda]}}{d_{L R}}\right)\left|\overline{\mathbf{D}}_{\mathrm{R}}\right|_{\lambda-j, \lambda-j}+\left|\overline{\mathbf{D}}_{\mathrm{R}}\right|_{\lambda-j, \lambda-j+1} .
\end{aligned}
$$

The determinant $\left|\bar{D}_{\mathrm{R}}\right|_{\lambda-j, \lambda-j-1}$ is obtained by removing the $(\lambda-j)$ - th row and the $(\lambda-j-1)-$ th column of $\left|\bar{D}_{\mathrm{R}}\right|$; the determinant $\left|\bar{D}_{\mathrm{R}}\right|_{\lambda-j, \lambda-j}$ is obtained by removing the $(\lambda-j)-$ th row and the $(\lambda-j)-$ th column; and the determinant $\left|\bar{D}_{\mathrm{R}}\right|_{\lambda-j, \lambda-j+1}$ is obtained by removing the $(\lambda-$ $j)$ - th row and the $(\lambda-j+1)-$ th column. The detailed expressions of the determinants $\left|\bar{D}_{\mathrm{R}}\right|_{\lambda-j, \lambda-j-1},\left|\bar{D}_{\mathrm{R}}\right|_{\lambda-j, \lambda-j}$, and $\left|\bar{D}_{\mathrm{R}}\right|_{\lambda-j, \lambda-j+1}$ are as follows:

$$
\begin{aligned}
\left|\overline{\mathbf{D}}_{R}\right|_{\lambda-j, \lambda-j-1} & =-\frac{d_{L R}^{[\lambda]}}{d_{L R}} \frac{\sinh (\lambda-j-1) \mu \cosh \left(N_{P}-\lambda\right) \mu}{\sinh \mu}, \\
\left|\overline{\mathbf{D}}_{R}\right|_{\lambda-j, \lambda-j} & =\cosh (N-\lambda) \mu\left[\left(\cosh \mu-\frac{d_{L L}^{[\lambda]}}{d_{L R}}\right) \frac{\sinh (\lambda-j-1) \mu}{\sinh \mu}-\frac{\sinh (\lambda-j-2) \mu}{\sinh \mu}\right], \\
\left|\overline{\mathbf{D}}_{R}\right|_{\lambda-j, \lambda-j+1} & =-\cosh (N-\lambda-1) \mu\left[\left(\cosh \mu-\frac{d_{L L}^{[\lambda]}}{d_{L R}}\right) \frac{\sinh (\lambda-j-1) \mu}{\sinh \mu}-\frac{\sinh (\lambda-j-2) \mu}{\sinh \mu}\right] .
\end{aligned}
$$

\section{Data Availability}

The data used to support the findings of this study are included within the article.

\section{Conflicts of Interest}

The authors declare that there are no conflicts of interest regarding the publication of this paper.

\section{Acknowledgments}

This work was supported by the National Natural Science Foundation of China (Grant no. 51838006).

\section{References}

[1] J. A. Haringx, "On highly compressible helical springs and rubber rods, and their application for vibration-free mountings, III," Philips Research Reports, vol. 4, pp. 206-220, 1949.

[2] J. M. Kelly, Earthquake-resistant Design with Rubber, Springer-, New York, NY, UK, 1997.

[3] J. Kelly and M. R. Marsico, "Stability and post-buckling behavior in nonbolted elastomeric isolators," Seismic Isolation and Protective Systems, vol. 1, no. 1, pp. 41-54, 2010.
[4] C.-H. Chang, "Modeling of laminated rubber bearings using an analytical stiffness matrix," International Journal of Solids and Structures, vol. 39, no. 24, pp. 6055-6078, 2002.

[5] A. N. Gent, "Elastic stability of rubber compression springs," Archive: Journal of Mechanical Engineering Science 1959-1982 (vols 1-23), vol. 6, no. 4, pp. 318-326, 1964.

[6] J. F. Stanton, G. Scroggins, A. W. Taylor, and C. W. Roeder, "Stability of laminated elastomeric bearings," Journal of Engineering Mechanics, vol. 116, no. 6, pp. 1351-1371, 1990.

[7] M. Imbimbo and J. M. Kelly, "Stability of isolators at large horizontal displacements," Earthquake Spectra, vol. 13, no. 3, pp. 415-430, 1997.

[8] M. Imbimbo and J. M. Kelly, "Stability aspects of elastomeric isolators," Earthquake Spectra, vol. 13, no. 3, pp. 431-449, 1997.

[9] C. G. Koh and J. M. Kelly, "A simple mechanical model for elastomeric bearings used in base isolation," International Journal of Mechanical Sciences, vol. 30, no. 12, pp. 933-943, 1988.

[10] I. Buckle, S. Nagarajaiah, and K. Ferrell, "Stability of elastomeric isolation bearings: experimental study," Journal of Structural Engineering, vol. 128, no. 1, pp. 3-11, 2002.

[11] K. N. Kalfas, S. A. Mitoulis, and D. Konstantinidis, "Influence of steel reinforcement on the performance of elastomeric bearings," Journal of Structural Engineering, vol. 146, no. 10, Article ID 04020195, 2020.

[12] K. N. Kalfas and S. A. Mitoulis, "Performance of steel-laminated rubber bearings subjected to combinations of axial 
loads and shear strains," Procedia engineering, vol. 199, pp. 2979-2984, 2017.

[13] L. Ding and H. P. Zhu, "Seismic response and vibration transmission characteristics of laminated rubber bearings with a single disorder," Journal of Engineering Mechanics, vol. 154, no. 12, Article ID 4019093, 2019.

[14] L. Ding, H.-P. Zhu, and L. Wu, "Analysis of mechanical properties of laminated rubber bearings based on transfer matrix method," Composite Structures, vol. 159, pp. 390-396, 2017.

[15] E. Takaoka, "Nonlinear mechanical model for laminated rubber bearings subjected to monotonic loading based on Haringx's theory," Journal of Structural and Construction Engineering, vol. 79, no. 701, pp. 913-921, 2014.

[16] H. P. Zhu and J. X. Tang, "Vibrational transmission characteristics of laminated rubber bearing," Engineering $\mathrm{Me}$ chanics, vol. 12, no. 4, pp. 109-114, 1995.

[17] H. Zhu and M. Wu, "The characteristic receptance method for damage detection in large mono-coupled periodic structures," Journal of Sound and Vibration, vol. 251, no. 2, pp. 241-259, 2002.

[18] T. Yin and M. L. Yin, "Damage detection in large periodicallysupported structures based on the characteristic receptance method and the sensitivity-based approach," Journal of Vibration and Shock, vol. 36, no. 22, pp. 93-99, 2017.

[19] H. P. Zhu and Y. Xu, "Damage detection of mono-coupled periodic structures based on sensitivity analysis of modal parameters," Journal of Sound and Vibration, vol. 285, no. 1-2, pp. 365-390, 2005.

[20] S. W. Doebling, C. R. Farrar, M. B. Prime, and D. W. Shevitz, "Damage Identification and Health Monitoring of Structural and Mechanical Systems from Changes in Their Vibration Characteristics: A Literature Review," LA-13070-MS, Los Alamos National Laboratory, UM, United States, 1996.

[21] W. D'Ambrogio and A. Fregolent, "The use of antiresonances for robust model updating," Journal of Sound and Vibration, vol. 236, no. 2, pp. 227-243, 2000.

[22] M. Dilena and A. Morassi, "The use of antiresonances for crack detection in beams," Journal of Sound and Vibration, vol. 276, no. 1-2, pp. 195-214, 2004.

[23] D. Wang, H. Zhu, C. Chen, and Y. Xia, "An impedance analysis for crack detection in the Timoshenko beam based on the anti-resonance technique," Acta Mechanica Solida Sinica, vol. 20, no. 3, pp. 228-235, 2007.

[24] D. Hanson, T. P. Waters, D. J. Thompson, R. B. Randall, and R. A. J. Ford, "The role of anti-resonance frequencies from operational modal analysis in finite element model updating," Mechanical Systems and Signal Processing, vol. 21, no. 1, pp. 74-97, 2007.

[25] J.-J. Sinou, "On the use of non-linear vibrations and the antiresonances of higher-order frequency response functions for crack detection in pipeline beam," Mechanics Research Communications, vol. 43, pp. 87-95, 2012.

[26] C. Hou and Y. Lu, "Experimental study of crack identification in thick beams with a cracked beam element model," Journal of Engineering Mechanics, vol. 143, no. 6, 2017.

[27] F. Wahl, G. Schmidt, and L. Forrai, "On the significance of antiresonance frequencies in experimental structural analysis," Journal of Sound and Vibration, vol. 219, no. 3, pp. 379-394, 1999.

[28] J. E. Mottershead, "On the zeros of structural frequency response functions and their sensitivities," Mechanical Systems and Signal Processing, vol. 12, no. 5, pp. 591-597, 1998.
[29] L. Mao and Y. Lu, "Selection of optimal artificial boundary condition (ABC) frequencies for structural damage identification," Journal of Sound and Vibration, vol. 374, pp. 245-259, 2016.

[30] Y. Zhang, H. P. Zhu, and S. Weng, "Detection in laminated rubber bearings based on wave propagation and sensitivity analysis," in Proceedings of the APFIS 7th Asia-Pacific Conference on FRP in Structures, Gold Coast, Australia, December 2019.

[31] L. Brillouin, Wave Propagation in Periodic Structures: Electric Filters and crystal Lattices, Dover Publications Inc., New York, NY, USA, 1946.

[32] D. M. Mead, "Wave propagation in continuous periodic structures: research contributions from Southampton, 19641995," Journal of Sound and Vibration, vol. 190, no. 3, pp. 495-524, 1996.

[33] X. Yong, J. H. Wen, D. L. Yu, and X. S. Wen, "Flexural wave propagation in beams with periodically attached vibration absorbers: band-gap behaviour and band formation mechanisms," Journal of Sound and Vibration, vol. 332, pp. 867-893, 2013.

[34] D. Bouzit and C. Pierre, "Wave localization and conversion phenomena in multi-coupled multi-span beams," Chaos, Solitons \& Fractals, vol. 11, no. 10, pp. 1575-1596, 2000.

[35] D. J. Mead, "Wave propagation and natural modes in periodic systems: I. Mono-coupled systems," Journal of Sound and Vibration, vol. 40, no. 1, pp. 1-18, 1975.

[36] G. H. Golub and C. Van Loan, Matrix Computations, Johns Hopkins University Press, Baltimore, 1983.

[37] A. M. Bruckstein, M. Elad, and M. Zibulevsky, "On the uniqueness of nonnegative sparse solutions to underdetermined systems of equations," IEEE Transactions on Information Theory, vol. 54, no. 11, pp. 4813-4820, 2008. 